Itinéraires Itinéraires

Littérature, textes, cultures

2016-1 | 2016

Écrire et créer avec les villes en mouvement

\title{
L'hypothèse de la traduction artiviste : mise en chantier indisciplinaire
}

The Hypothesis of Artivism as a Form of Translation. An Indisciplinary

Perspective

\section{Myriam Suchet et Sarah Mekdjian}

\section{(2) OpenEdition}

Journals

Édition électronique

URL : http://journals.openedition.org/itineraires/3312

DOI : $10.4000 /$ itineraires.3312

ISSN : 2427-920X

Éditeur

Pléiade

Référence électronique

Myriam Suchet et Sarah Mekdjian, «L'hypothèse de la traduction artiviste : mise en chantier indisciplinaire », Itinéraires [En ligne], 2016-1 | 2016, mis en ligne le 01 décembre 2016, consulté le 20 avril 2019. URL : http://journals.openedition.org/itineraires/3312 ; DOI : 10.4000/itineraires.3312

Ce document a été généré automatiquement le 20 avril 2019.

\section{$(1) \Theta \Theta$}

Itinéraires est mis à disposition selon les termes de la licence Creative Commons Attribution - Pas d'Utilisation Commerciale - Pas de Modification 4.0 International. 


\section{L'hypothèse de la traduction artiviste : mise en chantier indisciplinaire}

The Hypothesis of Artivism as a Form of Translation. An Indisciplinary

Perspective

Myriam Suchet et Sarah Mekdjian

Je vous construirai une ville avec des loques, moi. Je vous construirai sans plan et sans ciment un édifice que vous ne détruirez pas.

Henri Michaux, « Contre! ", La Nuit remue, Euvres complètes I, Paris, Gallimard, coll. « Bibliothèque de

la Pléiade », 1998, p. 457-458.

La langue de l'Europe, c'est la traduction. Mieux encore c'est la réalité des pratiques sociales de traduction à différents niveaux, le medium de communication dont tous les autres dépendent.

Étienne Balibar, Nous, citoyens d'Europe? Les frontières, l'État, le peuple, Paris, La Découverte, 2001, p. 316-317.

1 La ville - toute ville - peut se lire comme un ensemble de signes. Certains de ces signes sont métaphoriques, mais la plupart sont extrêmement littéraux et matériellement concrets : panneaux, enseignes, encarts et autres graffitis offrent en permanence un double langage verbal et graphique à déchiffrer. On ne s'étonne donc guère de voir la traduction occuper une place visible à l'œil nu dans les sémiotiques urbaines. Au Québec, les panneaux de signalisation "stop » ont été remplacés par des panneaux " arrêt » dans la foulée de la loi 101 (Charte de la langue française de 1977) et des décrets du gouvernement de René Lévesque. Le ministère des Transports a pourtant renversé cette décision en 1992, en alléguant que «stop » est considéré comme un mot français par l'Office québécois de la langue française depuis 1976. L'utilisation des termes «ARRÊT » 
ou « STOP » est donc aujourd'hui conforme à la réglementation - à condition que les deux termes ne figurent pas conjointement, car le Québec est officiellement unilingue. Dans d'autres provinces, en Ontario ou au Nunavut par exemple, le même panneau peut comporter plusieurs termes dans des langues différentes et donc traduire l'indication en français, en anglais, en cri ou en inuktikut. L'image ci-dessous montre un panneau bilingue en français et innu-aimun (langue de la Première Nation des Innus, longtemps appelés Montagnais) :

Fig. 1. Panneau « arrêt » bilingue en français et innu-aimun

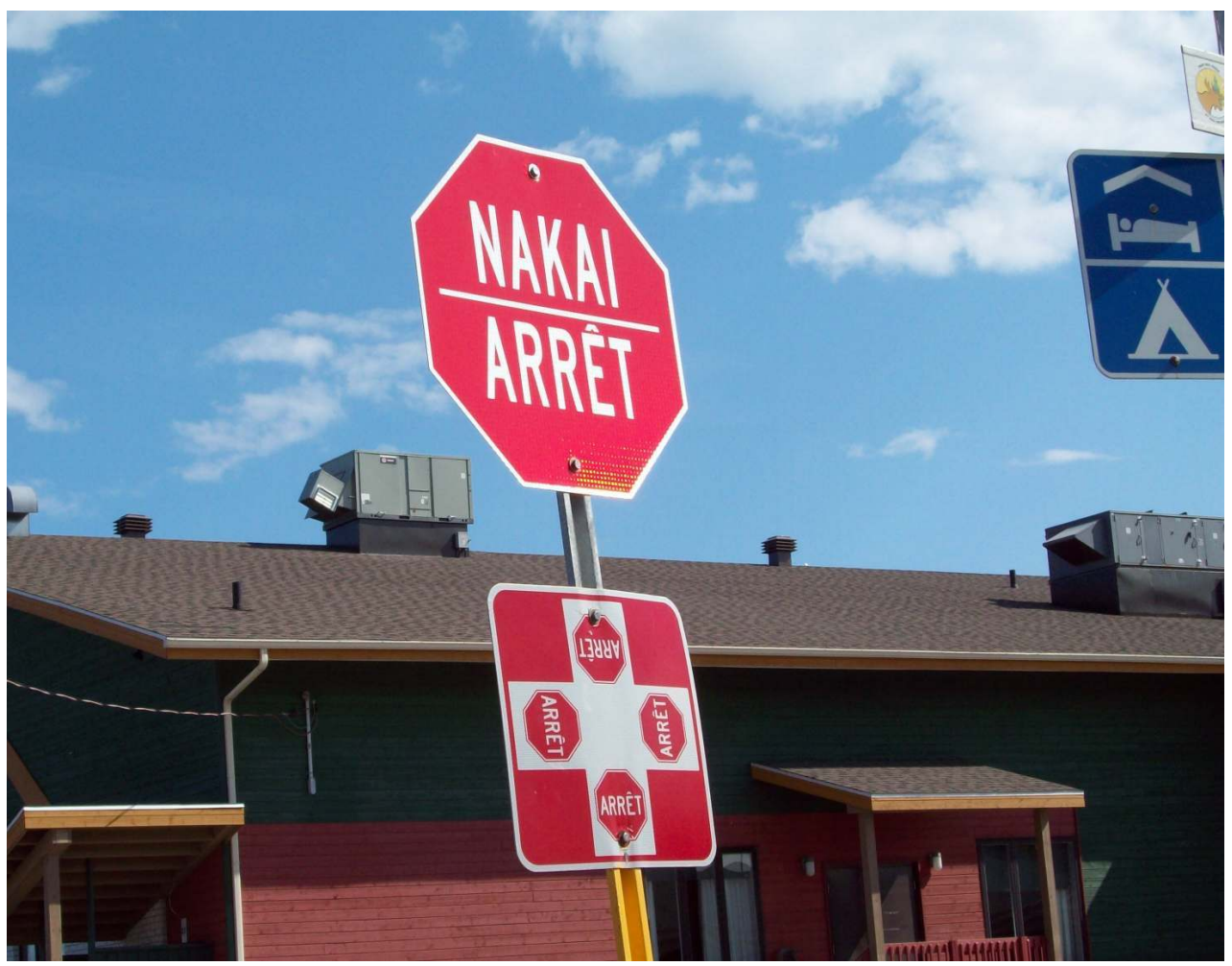

(C) Myriam Suchet.

Traduire (ou ne pas traduire) les signes urbains relève donc d'une décision politique qui inscrit les enjeux de pouvoir dans l'espace même de la ville. De Shanghai à Nicosie en passant par Bruxelles, Johannesburg ou Pula, c'est une histoire mondiale des rapports de force qui se donne à lire selon que les traductions opèrent ou n'opèrent pas. Cette histoire des grands conflits et des réconciliations spectaculaires n'est pourtant pas la seule à s'écrire dans la traduction urbaine. En marge des traductions qui opèrent à la manière de bras de fer entre des langues co-extensives à des communautés imaginées (nationales ou autres, cf. Anderson 1983), d'autres pratiques traduisantes investissent les espaces urbains et transforment en signes (ou en promesses) de potentialités nouvelles des espaces qui, jusque-là, n'apparaissaient même pas. Les interventions artistiques qui visent à transformer l'espace urbain, que nous qualifierons d'« artivistes » (Lindgaard 2005, Augoyard 2000, Douay 2012) mettent en jeu des formes de traduction qui sont souvent moins aisément repérables que celles mentionnées plus haut, peut-être parce qu'elles ne reconduisent pas les jeux de pouvoir habituels. Au lieu de rejouer des lignes de front déjà connues, elles détournent les attendus et les attentes - y compris en ce qui concerne la définition même de «traduction ». 
3 La traduction, qui n'appartient pas à l'arsenal théorique traditionnel des géographes ni des urbanistes, peut permettre d'articuler différemment la réflexion relative à la portée critique des interventions artistiques urbaines. D'où notre hypothèse : il serait possible d'envisager l'acte de "traduire " de sorte à penser à la fois ce qui se passe lors de certaines interventions décalantes dans l'espace de la ville (plan de l'objet analysé) et nos manières d'analyser ces reconfigurations (méta-plan de l'analyse elle-même). L'hypothèse de la traduction, pour le dire autrement, consiste à envisager les œuvres comme autant de formes de traduction. Il s'agit, en retour, de comprendre comment (et si) ces œuvres peuvent contribuer à repenser ce que signifie « traduire ». La théorie de la traduction est envisagée depuis longtemps par des universitaires dans les domaines de la (socio)linguistique et de la littérature, notamment chez celles et ceux qui s'intéressent aux contextes urbains (Simon 2007 et 2008). La prise en compte de la traduction n'intervient pas exclusivement au niveau des objets étudiés : elle engage souvent une démarche qui invite à sortir de l'institution de l'Université pour s'inscrire plus résolument dans la cité, où s'élaborent des initiatives de traduction non académiques. Nous pensons, par exemple, à deux initiatives déployées à Montréal lors du Printemps érable: le site internet Translating the Printemps érable et l'équipe tactique de traduction d'urgence Red Squad. Dans les deux cas, universitaires et non-universitaires ont travaillé ensemble en tant qu'interprètes et traductrices comme une manière de prendre part au mouvement de revendication né d'un conflit étudiant (contre la hausse des frais de scolarité) et grandi en lutte sociale (contre la marchandisation des biens communs, la langue de bois politique et la violence policière, notamment). Mentionnons encore le séminaire d'Esra Akcan: "Modernity in Translation», dans le cadre de l'exposition Actions: comment s'approprier la ville au Centre canadien d'architecture (CCA) du 26 novembre 2008 au 19 avril 2009, ayant donné lieu à la publication de l'ouvrage Architecture in Translation. Germany, Turkey and the Modern House (Akcan, 2012).

4 Cet article se propose d'amorcer une réflexion-pratique sur les formes urbaines d'artivisme en les envisageant à la croisée de trois approches : la théorie de la traduction (Myriam Suchet), la géographie urbaine critique (Sarah Mekdjian) et la reconfiguration à l'œuvre dans les dispositifs de recherche universitaire embrayés (c'est-à-dire impliqués dans une situation d'énonciation et de production qui excède les limites de l'institution). Une telle démarche peut être qualifiée d'indisciplinaire dans la mesure où elle s'efforce de tenir ensemble recherche, création et action. Il s'agit donc moins d'établir un pont entre deux disciplines données pour envisager les facettes complémentaires d'un même objet d'étude (comme le proposerait une approche inter- ou même transdisciplinaire), que de déstabiliser les expertises et les terminologies respectives de domaines spécialisés pour éprouver comment la pensée se trouve impactée dès qu'elle se préoccupe de son propre pouvoir de transformation. Pour le dire autrement: notre travail consiste à faire l'expérience de ce qui arrive à la réflexion quand elle se préoccupe d'avoir une action transformatrice, en commençant par faire retour sur ses propres protocoles de travail. La traduction nous servira de navette ou de médiateur pour opérer des passages entre concepts et imaginaires, pratiques et critiques.

5 Notre point de départ reste proche de notre zone de confort disciplinaire, du moins en termes de méthode : nous avons simplement changé de corpus en nous intéressant non à des textes littéraires ni à des espaces géographiques, mais à des initiatives artivistes urbaines pour essayer de les organiser selon une typologie (I, par Myriam Suchet et Sarah Mekdjian). Nous avons ensuite fait retour sur le terme même de traduction repensé, à 
partir de cette exploration des œuvres, en termes d'intervalle ou d'interstice (II, par Myriam). Le troisième et dernier temps de cette étude mobilise cette redéfinition et redistribue les cartes en proposant une analyse d'une pratique impliquée (par Sarah, en III) : les ateliers de cartographiques créatives et expérimentales mises en œuvre à Grenoble en 2013.

\section{Typologie exploratoire des formes de traduction dans l'art urbain contemporain}

6 Voyageant fréquemment d'une ville à une autre (notamment en Europe et en Amérique du Nord, terrains qui seront considérés ici de manière privilégiée), nous sommes frappées par le nombre et la diversité des performances et des œuvres qui expérimentent (c'est-àdire qui proposent en acte) d'autres manières d'habiter les espaces urbains et d'y vivre collectivement. Et puisque je (Myriam) voyage souvent avec, dans mes bagages, des réflexions sur la traduction, j'en viens à me demander: est-il possible et pertinent de considérer que ces formes artivistes mettent en jeu des opérations de traduction? Dans ce cas, de quel type de traduction s'agirait-il ? Pour tâter le terrain, nous choisissons de circonscrire notre corpus en utilisant une banque de données élaborée par le groupe de recherche "Présences du littéraire dans l'espace public canadien » dirigé par Marc André Brouillette, écrivain et professeur à l'université Concordia (Montréal, Canada): la plateforme PLEPUC.ORG. Le site recense plus de 600 œuvres canadiennes et se donne pour objectifs de :

- contribuer à la connaissance des rapports entre les diverses formes du littéraire et leurs modalités d'inscription dans l'espace public;

- favoriser la création d'œuvres en organisant des ateliers multidisciplinaires visant à renouveler les rapports entre l'individu et le littéraire ;

- diffuser des informations et des travaux qui puissent stimuler la réflexion sur les enjeux artistiques, esthétiques, sociaux, urbains gravitant autour de cette question. 


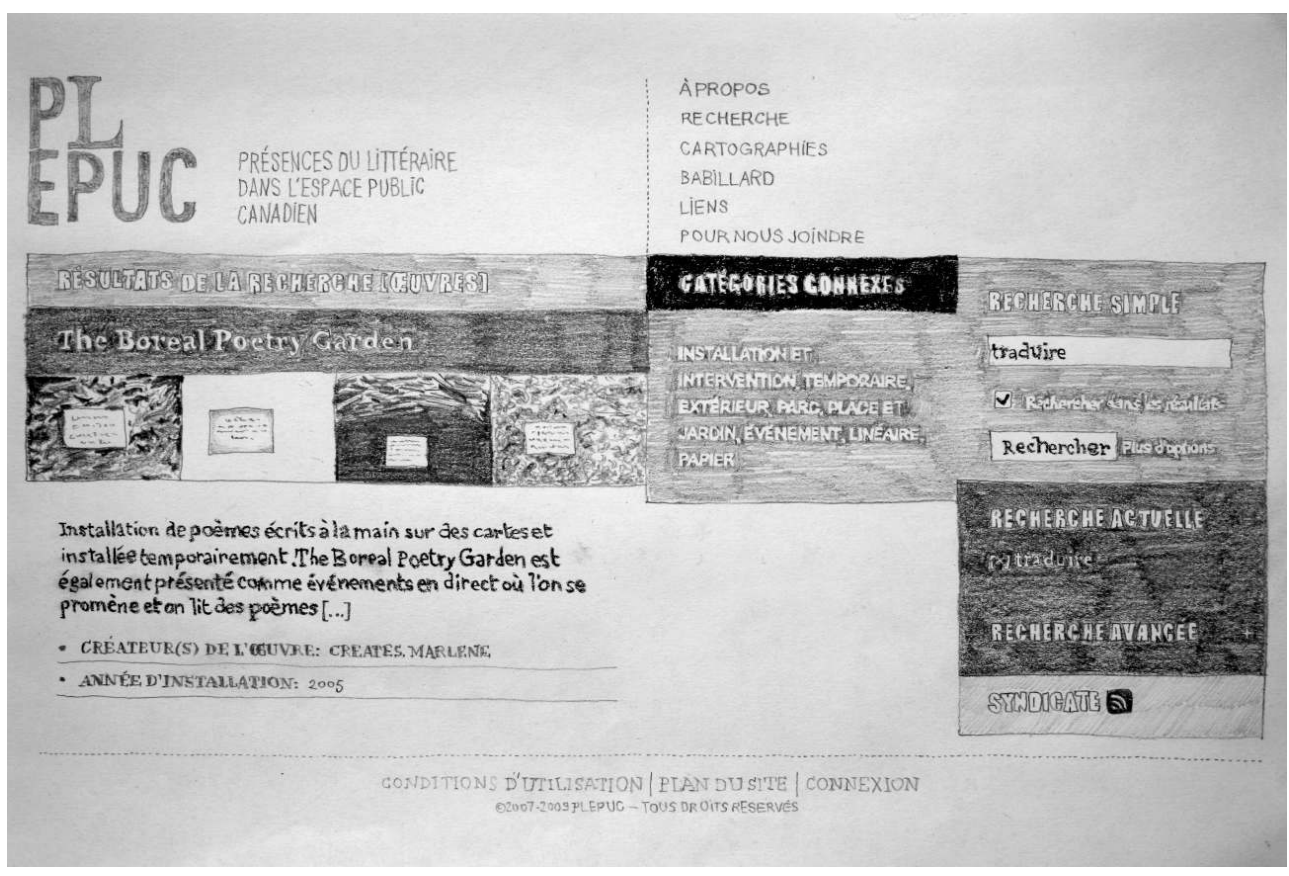

(C) David Elghozi.

7 En lançant deux recherches en français, l'une avec le mot-clé «traduction » et l'autre avec «traduire", on obtient six œuvres (cinq avec «traduction" et une avec «traduire »). C'est à partir de ces six résultats que nous avons décidé de travailler ${ }^{1}$. Afin d'éprouver notre hypothèse, nous commencerons par prendre au sérieux la possibilité d'envisager ces différentes œuvres, installations et performances comme autant de formes de traduction. En les échelonnant depuis les formes les plus littérales de traduction jusqu'aux pratiques les plus métaphoriques, on obtient une typologie en cinq catégories. Le principal intérêt de cette typologie étant de prendre appui sur l'exploration des œuvres pour aboutir, dans la deuxième partie, à une redéfinition de la traduction, nous ne saurions trop vous recommander de cliquer sur les hyperliens ou d'aller directement visiter la plateforme - nous profitons de cette remarque pour remercier les artistes qui nous ont accordé les droits pour reproduire leurs images !

\section{Installations traductionnelles stricto sensu en ville}

8 Certaines installations artistiques fonctionnent selon des opérations de (pseudo)traduction explicites, souvent pour jouer des décalages qu'une telle opération peut susciter.

9 C'est le cas, par exemple, du Berlin Project 2001 conçu par Rachelle Viader Knowles et réalisé avec trois artistes de Berlin (Allemagne) et trois artistes de Kitchener (Canada). Pour comprendre la pertinence de ce rapprochement, il faut se souvenir que la ville de Kitchener s'est appelée Berlin jusqu'en 1916. Dans les deux villes, les artistes ont d'abord joué le rôle de "guides " touristiques en racontant une histoire associée à un site urbain de leur choix. Cette histoire, racontée en allemand ou en anglais selon chaque artiste, a été enregistrée en format vidéo. Lors d'une deuxième phase du projet, les "guides » se sont transformés en «interprètes » : les vidéos ont donné lieu à des traductions parfois 
totalement fantaisistes, notamment lorsque l'interprète supposé ne connaissait pas la langue de départ. Lors de la dernière phase du projet, le montage des deux enregistrements a été installé dans la chambre des conseils de la mairie de Kitchener, en utilisant le réseau utilisé d'ordinaire pour les séances de travail.

Fig. 3. Rachelle Viader Knowles, Berlin Project 2001

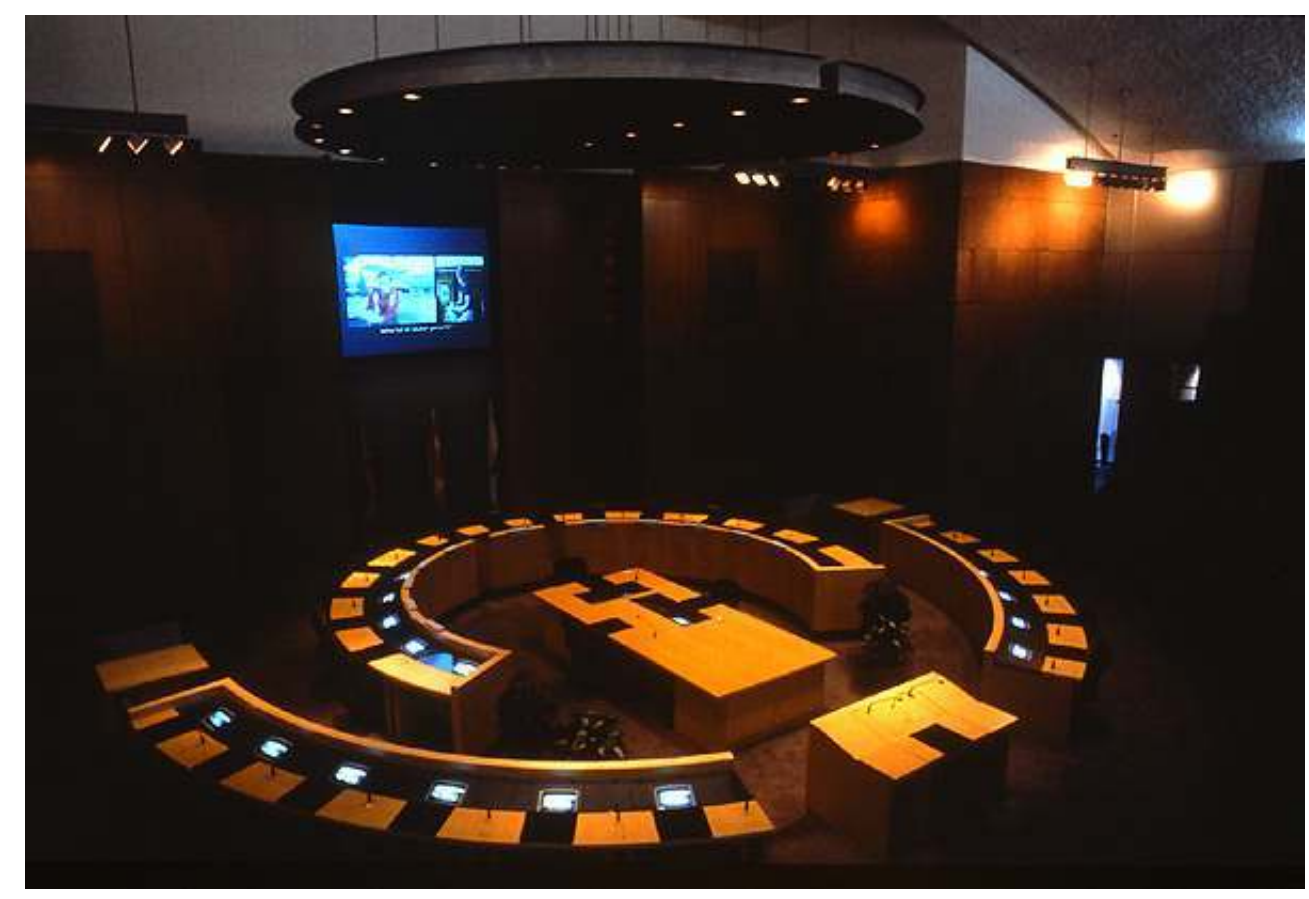

Fig. 4. Berlin Project 2001 (install)

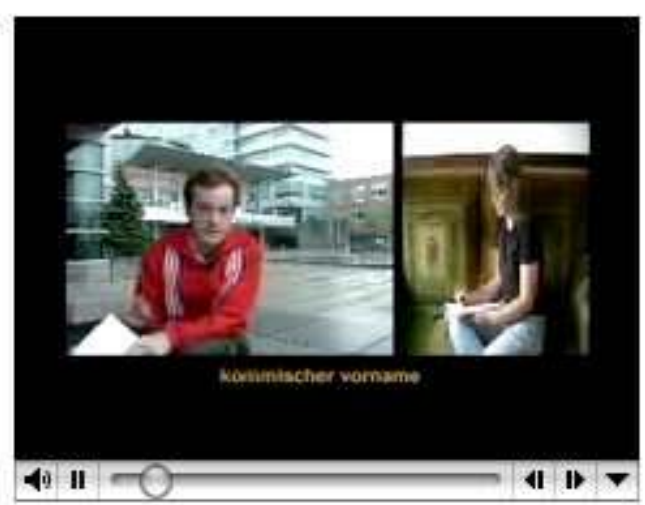

(c) Rachelle Viader Knowles.

10 La capture d'écran ci-dessus montre le dispositif vidéo, qui scinde l'écran en deux. Sur la partie gauche, on voit Ian Newton, un artiste visuel de Kitchener, qui raconte en anglais sa première visite dans cette ville. En regard, une artiste de Berlin prend des notes qui apparaissent comme des sous-titres en allemand. Au tout début de la vidéo, elle remarque que le lieu où se trouve Ian Newton ressemble à Alexanderplatz et il apparaît assez vite, à la lecture de ses commentaires, qu'elle ne comprend pas un mot d'anglais! Berlin Project 2001 met donc en œuvre la traduction à trois niveaux au moins. Le niveau le plus attendu est celui des langues (celles des histoires racontées et celles de leurs interprétations). 
Mais l'incompréhension fait du travail dit d'« interprétation» un véritable moment de création et de déploiement d'un imaginaire, et non un simple passage d'une langue à une autre. La traduction intervient aussi dans la mise en abyme des lieux, puisque Berlin se lit dans Kitchener et inversement. Enfin, la traduction opère dans le temps et la mémoire, puisque les histoires anciennes font ressurgir l'ancien toponyme de la ville de Kitchener. L'installation donne ainsi l'impression d'un effondrement de l'espace-temps : c'est Berlin (Allemagne) qui apparaît sur les moniteurs de la chambre des conseils de la mairie de Kitchener (Berlin).

11 Dans le cas du Berlin Project 2001, la traduction stricto sensu s'accompagne d'une série de déplacements qui ont pour conséquence la création d'une zone d'indétermination spatiotemporelle.

\section{Détournement de la signalétique (insurrection des signes urbains)}

12 La plateforme PLEPUC présente deux installations qui prennent la forme de panneaux publicitaires: une affiche réalisée par l'artiste Michael Fernandes où l'on peut lire «Inhabited by a spirit, worshipped by savages» (1991), et une photographie de Jamelie Hassan montrant le dôme coloré en faïence et le minaret de la mosquée Haidar Khan à Bagdad, en Irak. Cette photographie, qui date des années 1970, a été exposée lors de la guerre d'Irak en 1991 dans le cadre du programme In Control, organisé par l'agence Artcite, Windsor (commissaire : Lorenzo Buj). Dans ces deux cas, le mot «traduction » n'apparaît dans la notice que pour introduire la traduction en français des deux inscriptions : respectivement « un esprit l'habite, des sauvages le vénèrent » et «Parce que la ville de Bagdad existait et n'existait pas». La catégorie semble, néanmoins, suffisamment pertinente pour être maintenue. En effet, le détournement des signes urbains peut être considéré comme une forme de traduction même sans changement de langue, dans la mesure où un tel détournement révèle le fonctionnement sémiotique de la ville. Jean Baudrillard interprète les graffitis comme une "insurrection par les signes » dans son analyse de l'irruption des graffitis dans le New York des années 1970 (Baudrillard 1976:12) :

Irréductibles de par leur pauvreté même, ils résistent à toute interprétation, à toute connotation, et ils ne dénotent rien ni personne non plus: ni dénotation ni connotation. C'est ainsi qu'ils échappent au principe de signification, et en tant que signifiants vides, font irruption dans la sphère des signes pleins de la ville, qu'ils dissolvent par leur seule présence.

La plateforme PLEPUC offre deux autres exemples montréalais de détournement ou d'insurrection des signes urbains, qui n'apparaissant pas en cherchant «traduire» ni «traduction ». Le premier est celui de l'œuvre de Gilbert Boyer : Comme un poisson dans la ville. Il s'agit d'une installation de douze plaques disséminées sur différents édifices plus ou moins remarquables de la ville de Montréal. L'une de ces plaques, installée à l'angle de la rue Laurier et du boulevard Saint-Laurent, porte cette inscription :

QUELLE CHALEUR !

J'EN VIENS PRESQUE À RÊVER DE L'HIVER.

JE ME SUIS ARRÊTÉ PRÈS D'ICI.

UNE POUSSIÈRE DANS L'ÆIL.

À LA POINTE DE L'ANCIEN HÔTEL DE VILLE

SE TIENT EN ÉQUILIBRE UN OISEAU NOIR.

IL S'ÉVENTE LENTEMENT POUR SE RAFRAîcHIR.

LES AILES DEPLOYÉES. 
Fig. 5. Gilbert Boyer, Comme un poisson dans la ville

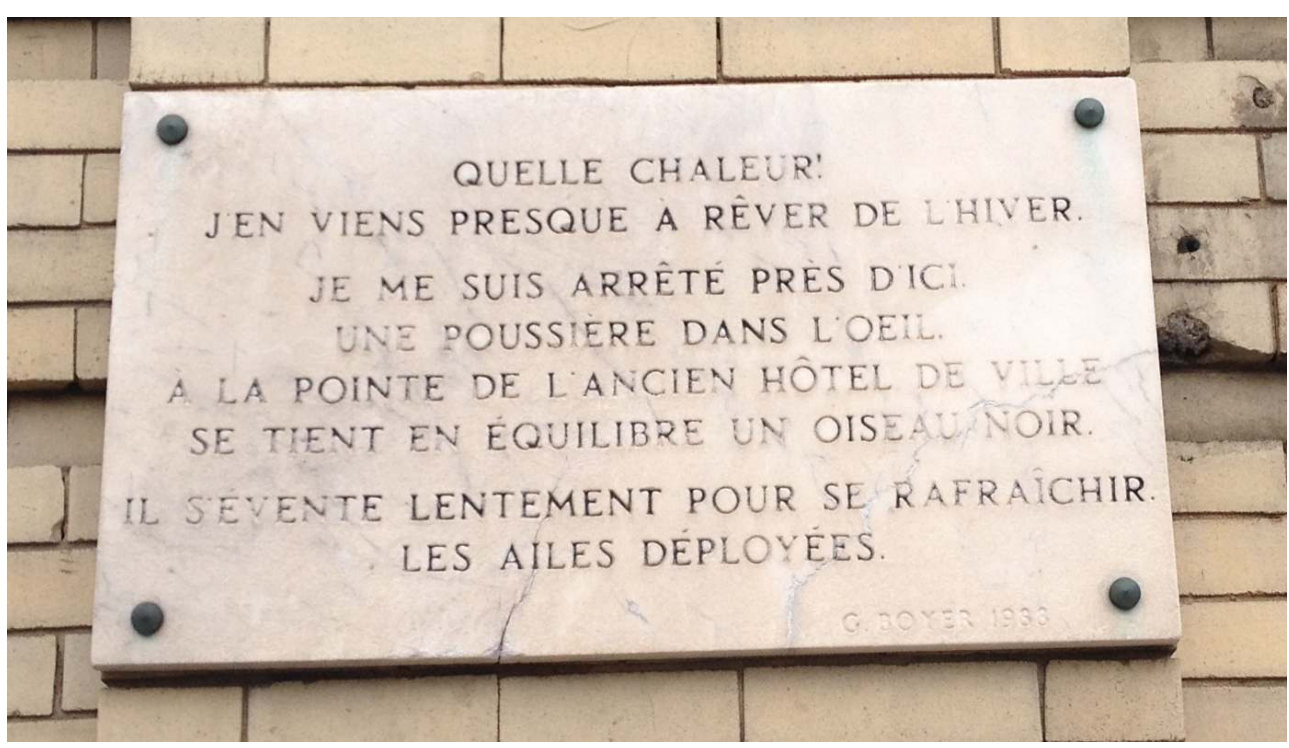

(c) Gilbert Boyer. Photographie Myriam Suchet.

$14 \mathrm{Au}$ lieu de monumentaliser la ville en officialisant son histoire, ces inscriptions consignent des notations éphémères. Elles invitent à poser un autre regard sur l'architecture et les vies qui l'habitent. La seconde œuvre répertoriée est celle de Roadsworth (nom d'artiste de Peter Gibson). Tandis que Gilbert Boyer détourne le fonctionnement codé des plaques commémoratives, Roadsworth détourne la signalétique du code de la route. Il intervient souvent à même le bitume, et bien qu'il travaille seul, il inscrit sa démarche dans une perspective collective et fondamentalement publique. Sur les œuvres répertoriées par le site, on peut voir les lignes blanches qui démarquent les emplacements de stationnement se métamorphoser en pissenlits géants soufflés par le vent, ou encore la ligne interrompue autorisant à doubler se transformer en tracé de découpage offert aux lames d'un gigantesque ciseau.

La traduction, ici, n'implique pas un changement de langue mais une transformation des signes eux-mêmes: la ville s'offre à un décodage inédit qui invite à la fois à la lire autrement et à enregistrer ses multiples manières, extrêmement concrètes et matérielles, de s'offrir à des pratiques de déchiffrement.

\section{Transformation ou création d'espaces (guerilla gardening)}

La troisième catégorie de notre typologie nous conduit dans des espaces moins visibles et moins fréquentés : des friches ou des bâtiments désaffectés qui se voient transformés en jardins, en voies cyclables, en espaces d'exposition ou de logement spontané, etc. Certaines de ces interventions sont pilotées par les politiques de la ville: on en trouve deux exemples sur le site de PLEPUc. Le plus souvent, cependant, ces installations de jardins ressortissent d'une ruse, d'une stratégie tactique plus ou moins illégale : on parle alors de guerrilla gardening (Reynolds 2008). Nous progresserons en allant du plus monumentale au moins visible, en nous demandant à chaque fois ce qui est engagé en termes de traduction. 
Un premier exemple très officiel est celui de la monumentale Fable VII de Trevor Gould, inspirée d'une fable de La Fontaine. Cette triple installation, située à l'Hôtel de ville d'Ottawa, se compose d'un lion, d'une inscription latine ("Ex Oriente Lux») et d'un bronze dans un bassin. Le terme qui nous intéresse apparaît dans la notice: "Traduction de l'inscription : "Ex Oriente Lux" (La lumière vient de l'est / Out of the East comes light). »

Fig. 6. Trevor Gould, Ex-oriente-lux.1

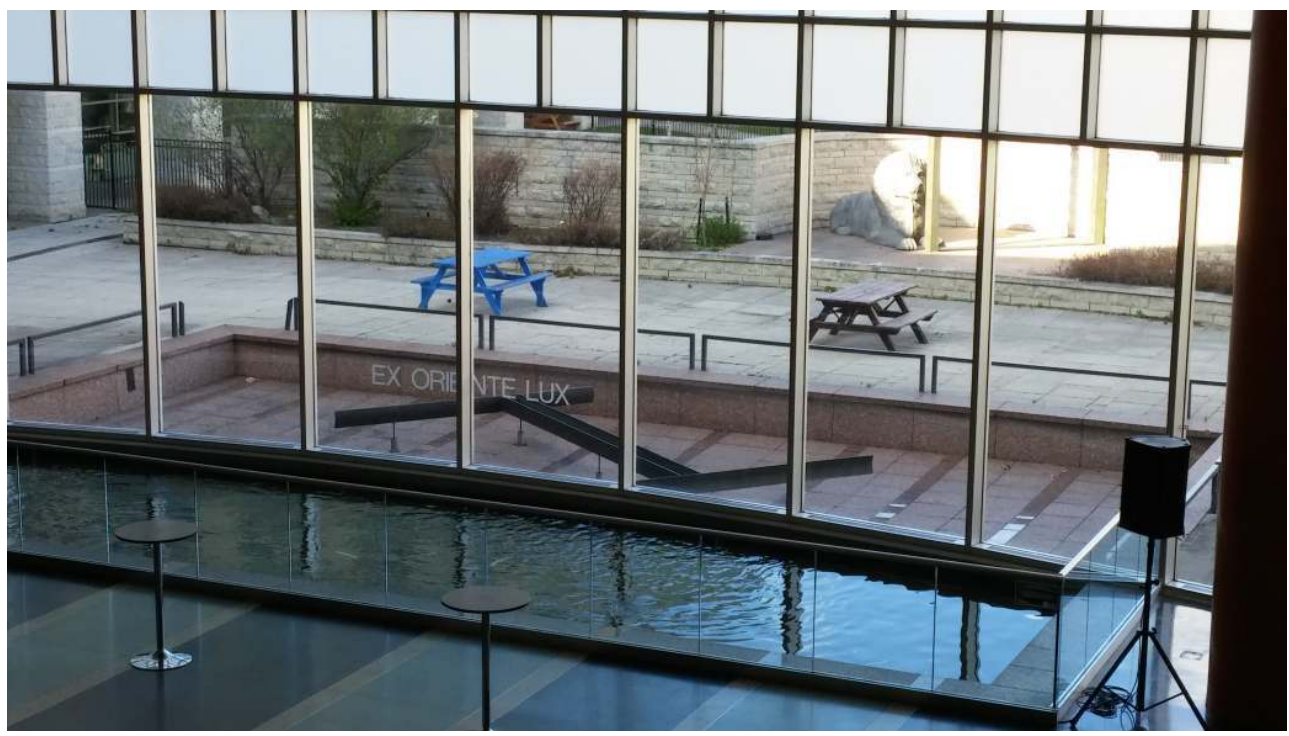

(c) Trevor Gould. Photographie Rainier Grutman.

Selon la notice toujours, "l'œuvre établit une action réciproque entre les espaces intérieur et extérieur, et crée un dialogue entre l'architecture des édifices avoisinants. Alors que le cadre de bronze tient lieu de cadran solaire, l'élément situé dans le bassin anime en permanence les alentours, présentant des notions de renouveau constant grâce à la lumière solaire et l'alignement directionnel ». Comme dans la catégorie précédente, la traduction n'implique ni coexistence ni substitution des langues : la citation latine n'est pas basculée en français. On notera tout de même que la numérotation de la fable de La Fontaine (ici « Fable VII ») varie selon la version: « La Cour du lion » est bien la septième dans la traduction anglaise parue chez Penguin, mais on la considère comme la sixième $\mathrm{du}$ livre VII en français. Reste que traduire, ici, consiste surtout à instaurer une dynamique d'échanges, un canal de circulations entre extérieur et intérieur, naturel et ouvragé, statique et mouvant, etc. La dimension politique, occultée dans cette explication, est cependant manifeste: le lion sculpté de manière monumentale est métaphorique de l'Empire, tout comme la lumière scénarise un rapport de force entre Orient et Occident, tandis que le lieu même de l'installation (à l'Hôtel de ville) désigne la question du pouvoir et son inscription dans des lieux institutionnels. L'artiste, né et formé en Afrique du Sud avant d'arriver au Québec en 1987, explique sur le site de l'université Concordia où il est professeur :

I work from the insight that sculpture is a form of social material and that producing exhibitions is an aspect of cultural research. The very act of putting objects on exhibition is comprehended as the substance of installation practice itself, in both its cultural and social variations. Working out from the perspective of Zoos, Botanical gardens, and World exhibitions as representations of a "geography 
of exhibiting," these sites are explored in their relationship to the contemporary museum through the significance of their related display practices.

cadre du réaménagement de la place J.-E. Laforce (Montréal, à l'intersection des rues Berri et Viger) à l'occasion du vingtième anniversaire des relations de coopération entre les villes de Lyon et de Montréal. Deux jardins ont été créés : à Montréal, le jardin de Lyon , œuvre conjointe du sculpteur Jean-François Gavoty et du paysagiste Guerric Péré - et à Lyon le jardin de Montréal réalisé par une équipe montréalaise composée de Michel Goulet, sculpteur, Réal Lestage, urbaniste chez Daoust-Lestage, et Julie Saint-Arnaud, architecte du paysage chez Vlan. Ces jardins ont quelque chose à voir avec le Berlin Project 2001, puisqu'il s'agit là aussi d'une manière de brouiller les espaces. Mais la mise en œuvre diffère. Hétérotopique par excellence, le jardin semble fonctionner comme un sas de télétransportation, permettant de passer de Montréal à Lyon et inversement. Dans l'intervalle, on ne revient jamais exactement au même endroit : tout se passe comme si un nouvel espace avait été généré.

\section{Fig. 7. Jean-François Gavoty, Jardin de Lyon}

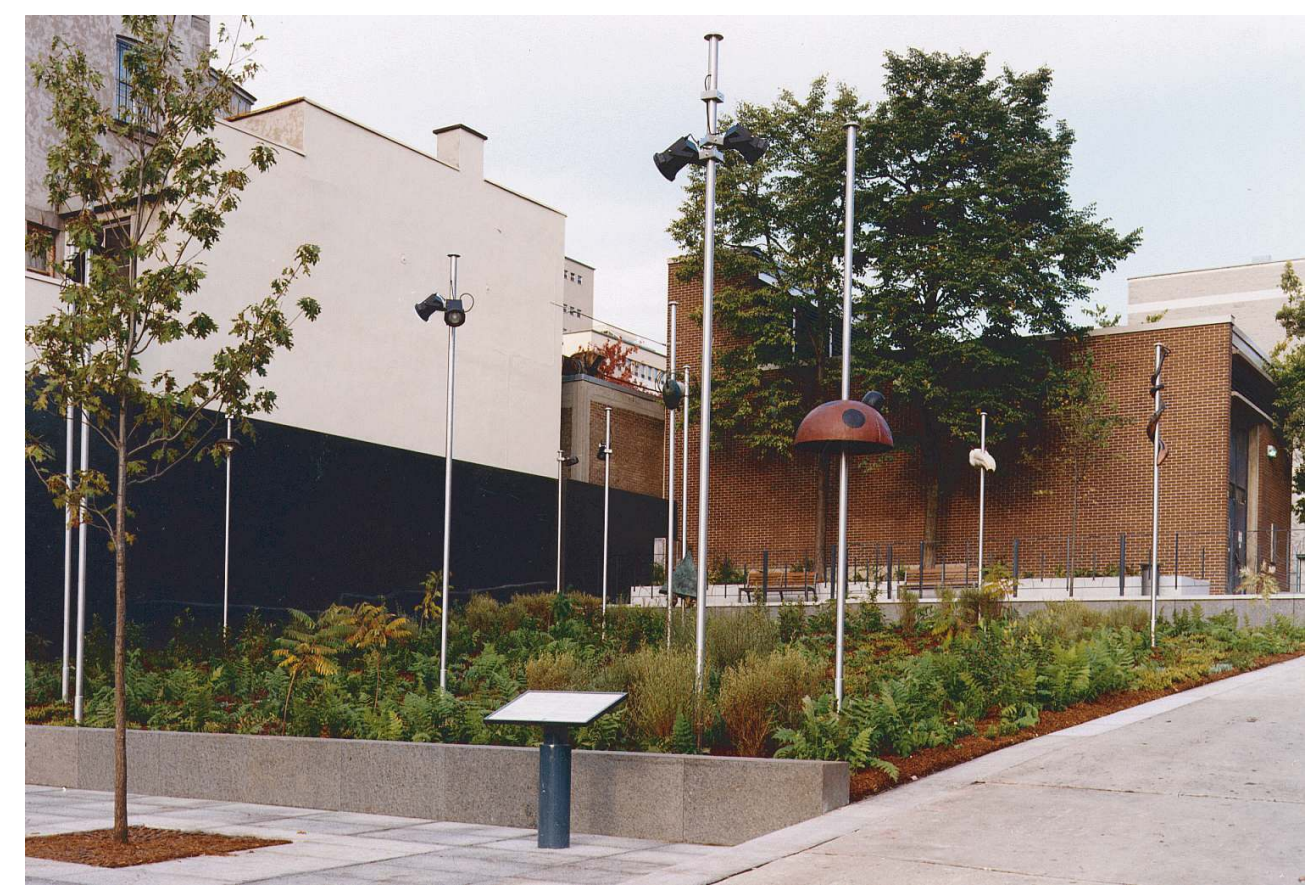

(C) Jean-François Gavoty.

Le site de l'artiste réserve une surprise qui semble confirmer notre hypothèse :

Les 6 sculptures en bronzes épinglées sont inspirées, avec plus ou moins de décalage, d'un alphabet de signes tracés par les vagabonds. Cette tradition très vive pendant la crise de 1929 s'est perpétuée dans les grandes villes américaines jusqu'à aujourd'hui. Terry Fox (1943-2008) en a réalisé le catalogue commenté ${ }^{2}$. 
Fig. 8. Jean-François Gavoty, Hoboes

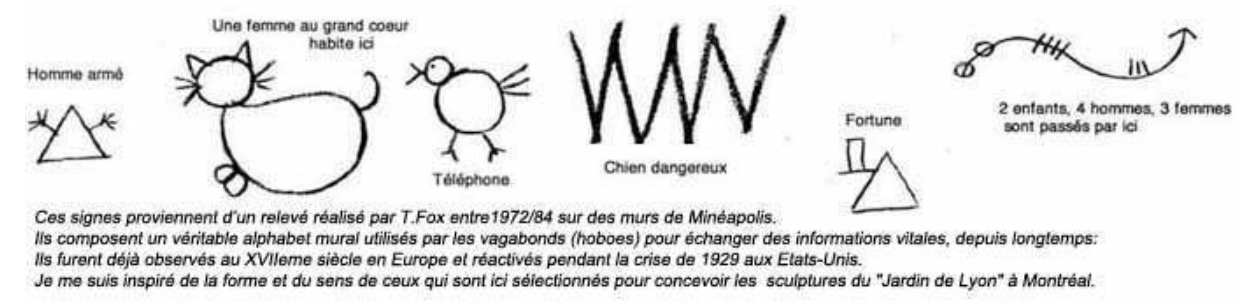

() Jean-François Gavoty.

Le jardin matérialise ainsi la translation d'un ensemble de signes empruntés ailleurs et traduits dans un espace qui ouvre un ailleurs au cœur même de la ville.

La seconde œuvre traductionnelle et jardinière présentée par le PLEPUC se différencie des précédentes en tirant le jardin hors de l'espace urbain, dans une œuvre autrement moins monumentale. Le Boreal Poetry Garden est une œuvre de Marlene Creates, composée de trois volets (précision offerte par l'artiste dans un courriel du 2 mai 2016): une installation éphémère de poèmes écrits à la main sur des cartons puis photographiés, une marche virtuelle (A Virtual Walk of The Boreal Poetry Garden) et un ensemble de performances in situ par des artistes (poètes, danseurs et musiciens) ainsi que des scientifiques (écologistes, chercheurs). Il s'agit donc, contrairement aux deux jardins mentionnés plus haut, d'une œuvre à durée limitée et installée à l'extérieur de la ville. C'est à Terre-Neuve, en effet, que prend place cette expérience poétique. Comme l'explique la notice :

Certains textes reflètent la vie du site géophysique et notamment des caractéristiques climatiques, la flore, la faune et l'histoire sociale, de même que l'expérience de l'artiste dans ce lieu. Pour l'artiste, l'emplacement des mots est fondamental pour traduire l'énergie rayonnante de leur sens et, bien sûr, leur beauté. 
Fig. 9. Marlene Creates, Boreal Poetry Garden

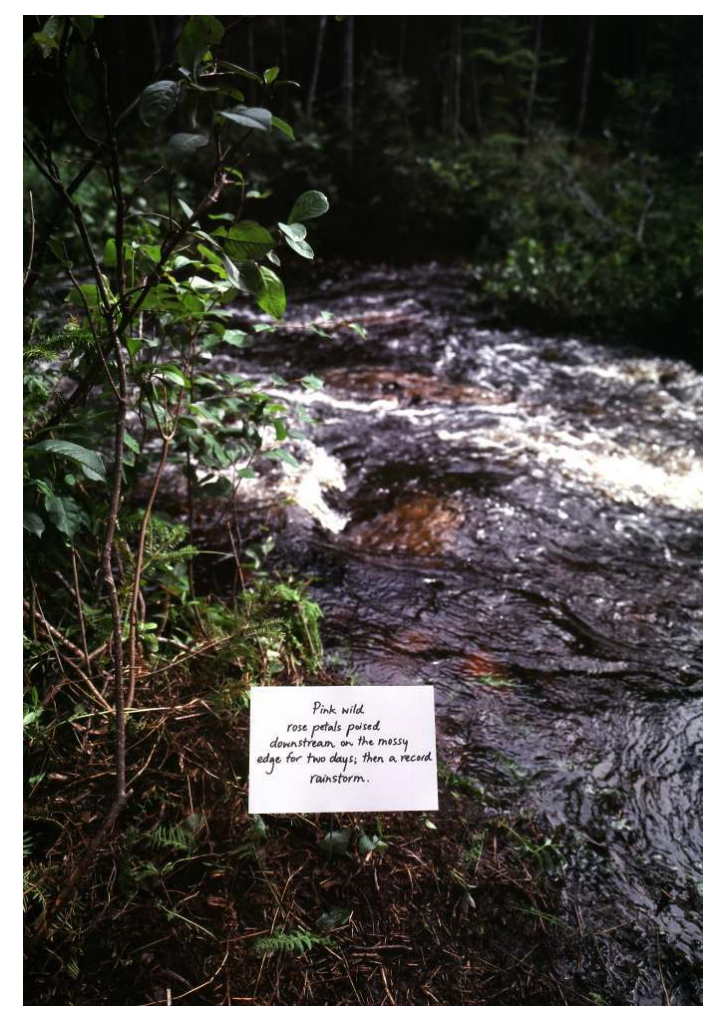

(C) MARLENE CREATES.

Le site personnel de l'artiste développe davantage la question linguistique : «One of the inspirations for the poetry garden is the rich Newfoundland vernacular. Its words reflect a very concrete correlation between this language and this landscape » et de donner en exemple : « Rub: a barely discernible passage through dense woods not used long or often enough to be a path. » Dans l'œuvre de l'artiste, qui a par ailleurs signé deux Language and Land Use (Alberta, 1993 et Newfoundland, 1994), « la langue » n'est pas dissociable du médium photographique ni du paysage qui, chacun à leur manière, font signe. Contrairement au projet de Parc de la langue française de Rober Racine dont le dispositif est proche, pourtant, l'enjeu semble moins de célébrer «la langue » et d'organiser un déplacement alphabétique exhaustif dans un dictionnaire spatialisé mais abstrait que de jouer du frottement physique entre les mots et leur lieu d'inscription. L'écart qui sépare le « parc » du « jardin » en est-il l'indice?

On pourrait sans doute davantage rapprocher ce travail de celui de Gilbert Boyer évoqué plus haut, Comme un poisson dans la ville, tant pour le détournement des signes attendus que pour le jeu avec les couches de mémoire et la manière de faire histoire. On observe néanmoins, chez Marlene Creates, deux déplacements supplémentaires: le premier consiste à extraire le texte d'un support à première vue "naturel » (une plaque sur un bâtiment, par exemple) pour, justement, l'installer en pleine nature. Le second consiste à prolonger l'œuvre éphémère par d'autres dispositifs: création photographique, performances artistiques et conférencières, etc. Comment ne pas voir là non seulement une forme de pratique traductionnelle mais aussi d'indiscipline? L'œuvre d'art devient l'œil d'un labyrinthe - ou l'étoile principale dans une constellation - d'expériences à la fois esthétiques et scientifiques. 
est pour suivre un brin ce fil indisciplinaire que nous franchissons le seuil de l'art institutionnel et quittons PLEPUC pour un instant, à la rencontre de groupes artivistes qui pratiquent une véritable guerrilla gardening. L'un des groupes encore actif est le CRAPAUD, Collectif de recherche sur l'aménagement paysager et l'agriculture urbaine durable issu de l'UQAM. Un autre groupe fameux, le Rebar, était l'organisateur du Park(ing) Day à San Francisco avec pour devise «Reclaim your city!» («Reprenez votre ville!»). Son mode d'action consistait à transformer, de manière éphémère et légale, une place de stationnement en un espace vert. Le collectif Rebar a déposé son concept par l'intermédiaire d'une licence Creative Common et permet de ce fait à des activistes du monde entier de se joindre à ce mouvement (Hou 2010). Mentionnons encore le collectif des Amis du Champ des Possibles, projet citoyen lié à un ancien site industriel situé dans le Mile-End à Montréal, au bord du chemin de fer. Le Champ des possibles, investi de longue date par les riverains, se constitue depuis quelques années en force de proposition urbaine sous l'impulsion combinée d'un auteur et naturaliste urbain, Roger Latour et d'une artiste, Emily Rose-Michaud.

Fig. 10. Le Champ des possibles

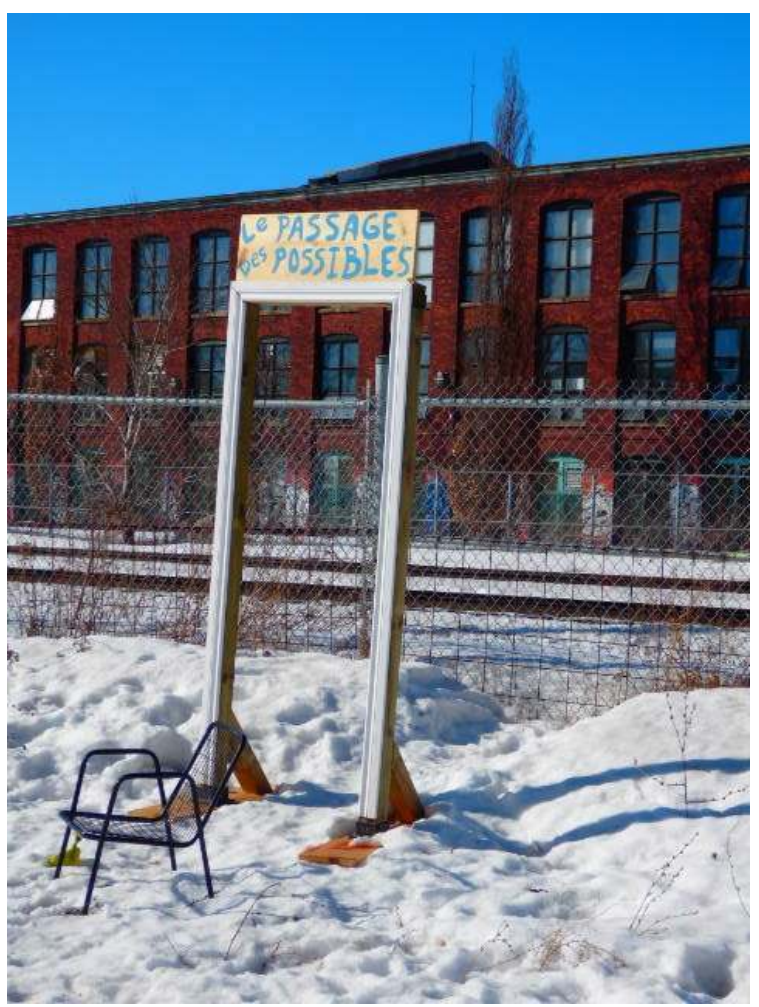

(c) Photographie Myriam Suchet.

es brèves évocations on retiendra surtout le caractère proliférant du geste d'investir ou de créer un jardin : davantage que d'autres œuvres ou actions, la guerilla guardening se conjugue volontiers au pluriel. Traduire devient, alors, une manière de faire collectif.

\section{Polyphonies inouïes et narrations invisibles}

La sixième œuvre répertoriée en termes de traduction sur la plateforme PLEPUC invite à ouvrir une dernière catégorie. Il s'agit d'une sculpture haute de plusieurs étages dans 
l'atrium du Broadway Campus Building, un immeuble du complexe Vancouver Community College. Réalisée par Antonia Hirsch, Double Blind donne à voir une monumentale traduction en braille de l'échelle d'acuité visuelle telle que la fixe le tableau de Snellen.

Fig. 11. Antonia Hirsch, Double Blind

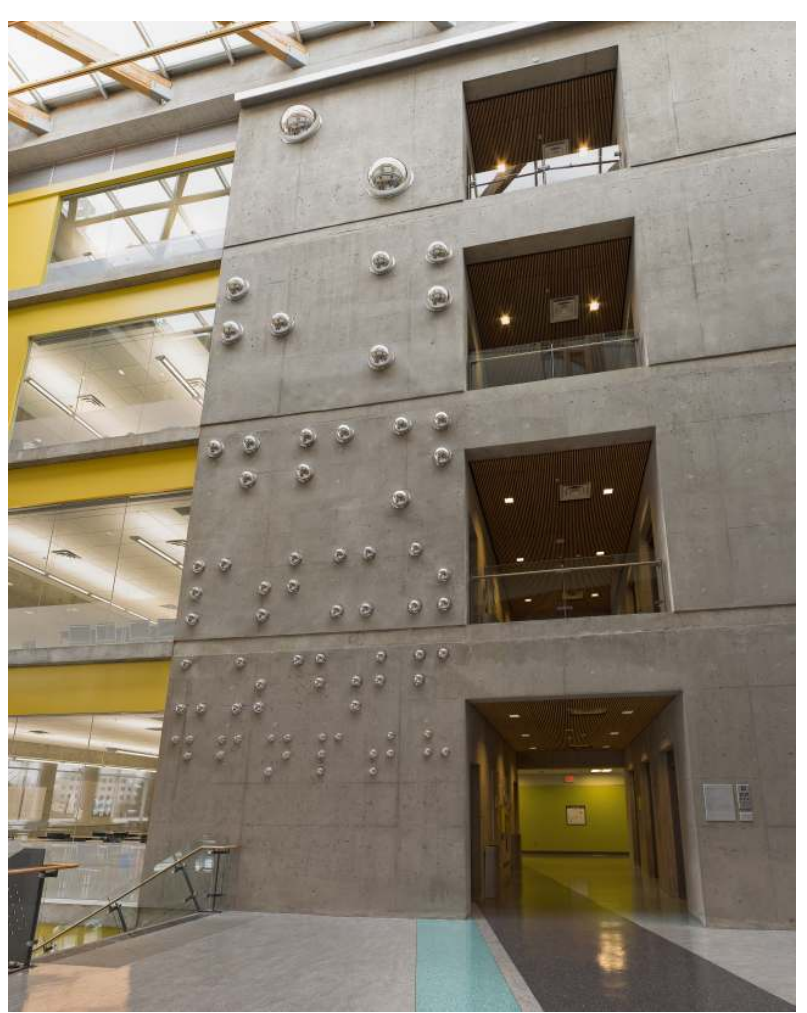

(C) ANTONIA HIRSCH.

Le titre de l'œuvre (Double Blind) en dévoile le message : dénoncer une double contrainte ( Double Bind) - ici celle de ne pas savoir lire le texte en braille quand on peut le voir et de ne pas pouvoir voir le texte quand on sait lire le braille. L'ironie a, cependant, une autre cible: l'espace public lui-même, qui s'organise à la manière d'un panoptique au sein duquel tout doit être visible en tout temps ${ }^{3}$. Faut-il considérer que la traduction de l'échelle d'acuité visuelle en braille vise à rendre visible un point aveugle ou, à l'inverse, qu'il s'agit d'inviter à se soustraire aux regards au beau milieu d'un lieu fait de glaces et de miroirs? Quoique par des chemins opposés, les deux interprétations convergent vers un travail de renversement des codes si établis qu'ils en sont devenus imperceptibles - et qu'ils invisibilisent les options et réalités alternatives. On pourrait dire, en passant de la vue à l'ouïe, qu'une telle installation vise à restituer des voix que l'histoire officielle condamne au silence ${ }^{4}$. Pour vérifier la pertinence de cette dernière catégorie et affiner sa caractérisation, nous avons cherché d'autres exemples d'une telle forme de traduction, qui consiste à rendre visible (ou audible).

Sacrée montagne est un projet de l'écrivaine et journaliste Hélène de Billy et du photographe Gilbert Duclos, chapeauté par l'Office national du film du Canada ${ }^{5}$. Il s'agit d'une flânerie interactive sur le Mont Royal, immense parc situé au cœur de Montréal. 
Fig. 12. Gilbert Duclos, Sacrée montagne (capture d'écran)

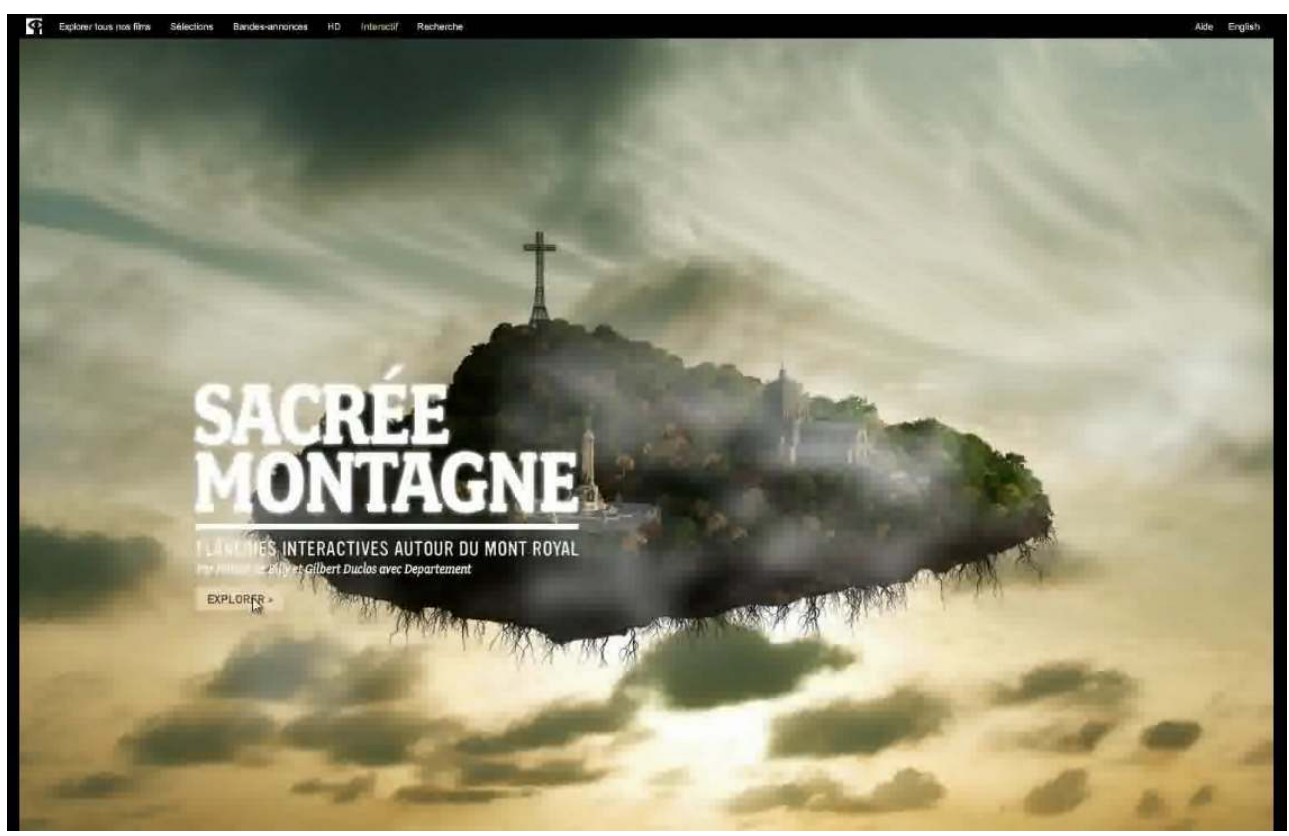

(c) Gilbert Duclos.

31 Six lieux ont été identifiés par les artistes pour articuler les six temps de la visite virtuelle : les cimetières, la croix, l'oratoire Saint-Joseph, la statue de la Renommée (dite statue de l'Ange), le lac des Castors et les sentiers du mont. À chacun des six lieux sélectionnés par les artistes sont associés des interfaces secondaires qui permettent d'accéder à des contenus variés : albums photos, courts-métrages, entrevues avec des personnalités québécoises, enregistrements d'événements culturels, courts documentaires sur des événements ayant marqué l'histoire récente du Mont Royal (par exemple, la canonisation en 2010 du Frère André), etc. Les liens menant à chacun de ces contenus sont placés autour d'une image virtuelle du monument ou du lieu choisi, activables en passant le curseur de la souris. 
Fig. 13. Gilbert Duclos, Sacrée montagne (photographie)

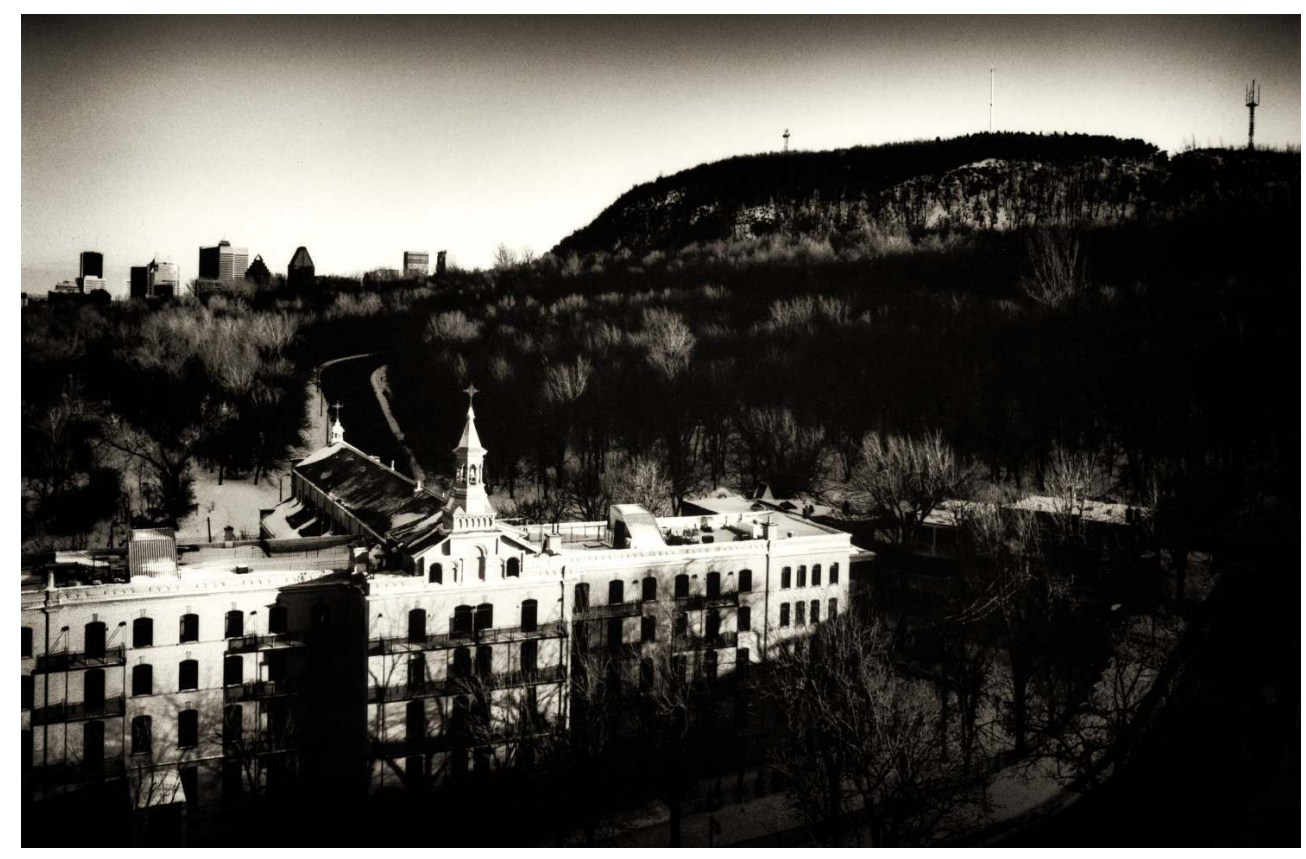

(c) Gilbert Duclos.

Si la catégorie précédente avait introduit l'idée d'une pratique collective, celle-ci va plus loin encore dans la dimension interactive. Il n'y a plus, dès lors, une artiste ou un créateur d'un côté et des usagers de l'autre mais, jusqu'à un certain point, des co-auteurs ou du moins des spectacteurs ou des « wreaders » (Landow 2006). L'invitation à la navigation ou à l'interaction, augmentée de la complexité ainsi induite, explique en partie l'importance prise par les cartes dans bon nombre de ces expérimentations. Le détournement des cartes conventionnelles peut s'apparenter à une narration alternative lorsqu'il consiste à explorer les points aveugles des représentations spatiales officielles de la ville. Ainsi, Kiss and tell de Stephen Foster se présente comme un plan de la ville de Québec où sont indiqués, en lieu et place des monuments officiels, les graffitis photographiés par l'artiste. Comme l'explique la notice du laboratoire nt2 qui répertorie cette œuvre :

Se promenant dans les rues de la ville de Québec et photographiant toutes ces traces de récits personnels, l'artiste, dont le travail s'intéresse principalement à la représentation des Autochtones dans la culture populaire, cherche à faire naître un espace de dialogue politique et social. Kiss and tell présente donc à l'internaute un rassemblement de photographies de graffitis intimement inscrits dans la cartographie de la capitale qu'il est invité à parcourir, tissant ainsi des liens entre toutes ces histoires personnelles inscrites dans la collectivité du lieu public. 
Fig. 14. Stephen Foster, Kiss and tell

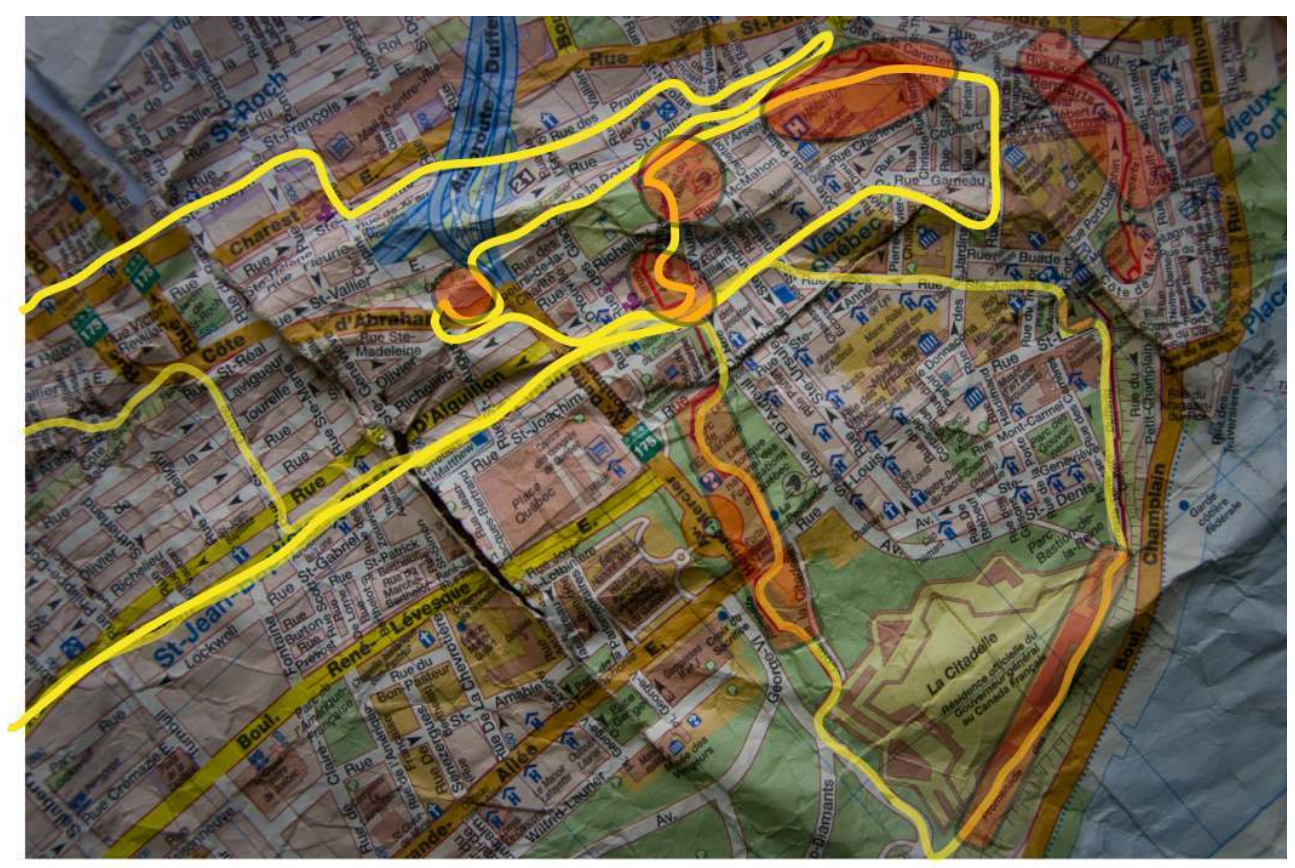

Main map interface for Kiss $N$ Tell by Web Artist Stephen Foster.

(C) STEPHEN FOSTER.

Un autre exemple absent du site de PLEPUC est le projet collectif Détours, produit et réalisé par l'Agence TOPO sous la direction de Taien Ng-Chan. Il s'agit de cartographier non pas des lieux mais des trajets, souvent en accompagnant les tracés visuels d'archives sonores. Taien Ng-Chan réalise, parmi d'autres projets, deux cartes : « Passages de ville » et « Les rues des saints ", où des vidéopoèmes invitent à suivre la route d'étrangers croisés dans des autobus. 
Fig. 15. Detours : Poetics of the City (capture d'écran), Taien Ng-Chan, Agence TOPO

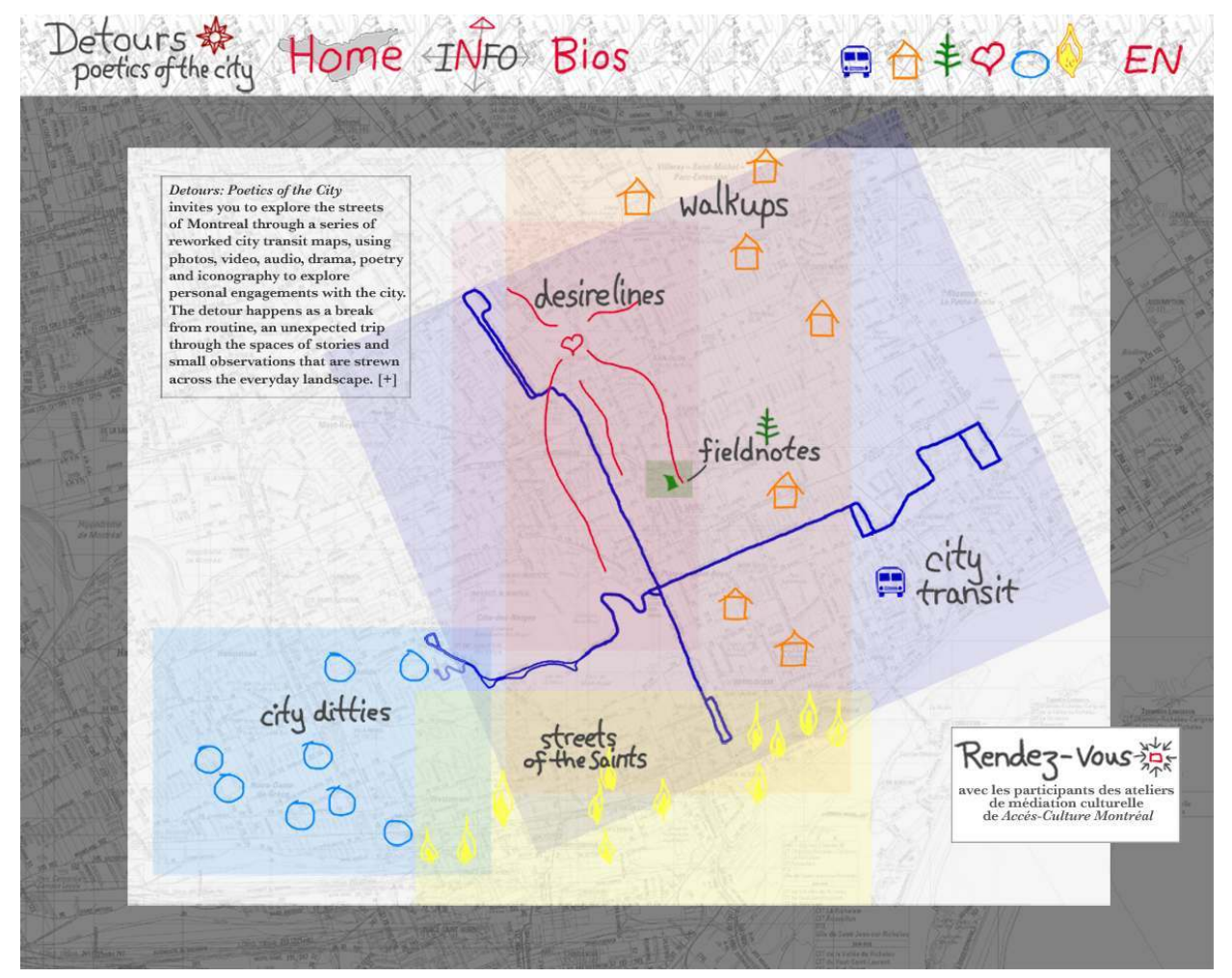

(C) TAIEN Ng-CHAN.

Le même collectif héberge aussi les Notes de terrain (Field Notes) d'Emilie O'Brien, qui explore le même « Champ des possibles » que celui mentionné dans la troisième catégorie (Transformation ou création d'espaces) avec des broderies, des «textworks », des images, et une entrevue avec Emily Rose Michaud.

Imaginée à partir des six œuvres trouvées en entrant « traduire » et « traduction » dans le moteur de recherche en français de la plateforme PLEPUC, cette typologie en quatre catégories ne laisse pas indemne notre représentation de la traduction. Il nous a semblé nécessaire, au terme de ce premier travail de catégorisation exploratoire, de faire retour sur le terme même de "traduction » afin d'évaluer comment il s'est déplacé, et en quels sens. Car si les œuvres envisagées ici sont vraiment, chacune à sa manière, des formes de traduction, alors "traduire" ne recouvre pas seulement ce que nous avons cru jusqu'alors.

\section{Un « interstice figural » : le battement du traduire urbain comme re-potentialisation du réel (Myriam Suchet)}

Quand je dis que la traduction me semble beaucoup avoir affaire aux énoncés, je parle de solitude. Parce que les énoncés sont rares. Pas rares parce qu'il y en a peu, mais parce qu'ils ne sont repérables que dans un espace raréfié, un no man's land, un espace sans sujet. [...] Terrain qui n'appartient pas. No man's land. Ni à vendre ni à bâtir. Et surtout pas terrain de rencontres, d'échanges, de dialogues, de discussions, 
d'influences, bref de communication. Mais un espace inaugural d'observation et de réflexion. (Hocquard 1997) consignées dans la typologie. Le point commun entre les œuvres répertoriées (qu'elles mettent en jeu des signes verbaux ou graphiques, des jardins ou des graffitis, des lieux architecturés et officiels ou marginaux et non bâtis, qu'elles prennent la forme de cartes, d'installation, de performances ou de jardins) consiste à ménager, pour un court instant, une autre dimension dans l'espace urbain. Il s'agit moins de la génération d'un nouvel espace que de la requalification d'un espace existant: c'est sur place et en quelque sorte sous nos pieds que l'œuvre transforme où nous sommes. Ces installations suggèrent donc de redéfinir la " traduction » comme une manière de changer la donne et de ré-ouvrir des horizons au-delà de la simple déclinaison des possibles déjà existants. Ces œuvres, en d'autres termes, traduisent dans la mesure où elles s'efforcent de proposer des situations et des actions qui ne préexistaient pas dans le répertoire des pratiques urbaines déjà constitué et disponible. On pourrait donc dire que la traduction opère dans la ville comme un battement. En termes « urbains ", on pourrait dire que la traduction ouvre des brèches, ou encore qu'elle fonctionne à la manière d'un interstice. Cet interstice ne trouve pas nécessairement d'inscription spatiale entre deux espaces existants : il peut être vertical ou orthogonal, à la manière d'une réalité augmentée qui n'est pas un ajout de matière mais une virtualisation ou re-potentialisation de celle-ci. Pour le dire autrement: la « réalité » de ce qui semblait indéniablement « être là » redevient une simple actualisation possible mais non nécessaire ni unique, ce qui ré-ouvre non seulement au possible (qui ne perturbe guère la "réalité ») mais au potentiel (qui redistribue les cartes de ce qui est et de ce qui pourrait être, cf. Lévy 1998). dans un article intitulé « Multiplicité interstitielle », où il explique :

Les interstices représentent ce qui résiste encore dans les métropoles, ce qui résiste aux emprises normatives et réglementaires, ce qui résiste à l'homogénéisation et à l'appropriation. Ils constituent en quelque sorte la réserve de « disponibilité » de la ville. Du fait de leur statut provisoire et incertain, les interstices laissent deviner ou entrevoir un autre processus de fabrication de la ville, ouvert et collaboratif, réactif et transversal. C'est effectivement sur ce plan-là, à la fois méthodologique et formatif, politique et heuristique, que se vérifie l'importance de l'expérience interstitielle. (Nicolas-Le Strat $2007: 118$ )

Pour Pascal Nicolas-Le Strat, les pratiques artistiques ont souvent un «rôle de dévoilement ou de révélateur de déploiement ou de dépliement de ces potentialités accumulées par une société devenue multitude ». Ce sont les œuvres qui opèrent le «travail interstitiel» (ou qui génèrent l'expérience interstitielle) permettant de « renouer avec ces hypothèses et ces devenirs disqualifiés par l'économie générale de la société, maintenus en lisière de son développement ou ensevelis sous la somme de ses productions marchandes » (Nicolas-Le Strat 2007 : 118).

Mais comment comprendre que la traduction puisse produire quelque chose comme un interstice sans réduire celui-ci à une simple figure, une image métaphorique? 
Paradoxalement, comprendre la traduction comme opératrice d'interstice suppose de basculer d'une représentation spatiale (le passage ou le transfert comme un vecteur conduisant de manière linéaire d'une "langue source" à une "langue cible») à une représentation temporelle disruptive et intempestive où l'acte de traduire interrompt le déroulé de la parole, dénaturalise les discours. Si l'interstice évoque une brèche, une faille, un intervalle dans l'espace saturé de la ville, il ne faut pas laisser sa dimension spatiale oblitérer sa dimension temporelle. Dans l'ouvrage collectif Quand la marge est créatrice, les interstices urbains initiateurs d'emploi, Hélène Hatzfeld, Marc Hatzfeld et Nadja Ringart insistent pour dire qu'il faut comprendre l'interstice dans une double dimension spatiale et temporelle :

Du point de vue spatial, il s'agit de rechercher ces morceaux de ville qui échappent aux régulations générales. On en trouve partout, depuis les terrains vagues jusqu'aux squats, en passant par les recoins cachés, les parkings, les endroits accaparés par divers types de marginalité. [...] Du point de vue temporel, les interstices renvoient au fait que la ville est en mouvement perpétuel, qu'elle évolue de façon plastique dans le temps. Des zones auxquelles était à une époque affectée une fonction précise perdent cette fonction à l'occasion de transformations économiques et sociales et deviennent tout à coup des endroits inutiles de la ville, et donc des poches qui favorisent l'apparition d'activités marginales. (H. et M. Hatzfeld, Ringart $1998: 36$ )

41 Les potentialités et la puissance de frayage de l'interstice tiennent ainsi en partie de son caractère éphémère - qui est aussi sa limite.

Basculée de l'axe spatial à l'axe temporel, la traduction quitte le référentiel de «la langue » et se reformule en discours. Autrement dit, la traduction telle qu'opérée par l'artivisme consiste moins à passer d'une langue à une autre qu'à rappeler que les langues sont, avant tout, des pratiques. Comme l'énonçait Meschonnic :

D'une langue, de toute langue, on n'a que des discours. C'est ce truisme qu'il faut quand même énoncer, quitte à braver le ridicule - mais le génie et la clarté française sont là pour rappeler que ce n'est pas inutile: que le mode d'existence d'une langue et le mode d'existence du discours sont radicalement différents. (Meschonnic 2000 : 31)

Et encore (Meschonnic 1997 : 32) :

La langue seule, du point de vue du nominalisme des discours, n'existe pas. Les mots, seuls, n'existent pas. Sauf dans le dictionnaire. En ce sens, pas plus qu'aucune langue, la langue française n'existe pas. Ce serait donc en termes d'énonciation qu'il faudrait rendre compte de la traduction. Traduire serait l'une des modalités permettant de s'extraire du "ronron» discursif quotidien pour frayer la voie à des paroles inédites.

La jonction entre le battement urbain de la traduction artiviste et sa puissance énonciative subversive peut être facilitée en rapprochant la notion d'interstice de celle du figural, développée dans un tout autre champ par Laurent Jenny. Stylisticien en analyse du discours, Laurent Jenny s'est intéressé à décrire cet événement qu'est l'énonciation. Il suggère de "penser à un énoncé comme à une "donne", un coup de dés lexical » (Jenny 1990 : 16). Tandis que le jeu quotidien du langage ne bouleverse guère les règles de ce jeu, la littérature, elle, transforme jusqu'aux conditions de possibilité du lancer de dés - c'est ce que Laurent Jenny appelle le figural (20):

Le figural, tout en ayant pour fond l'événementialité discursive, contraint à reconsidérer la forme même de la langue (son découpage en signes, ses règles combinatoires), il nous ramène à une décision "première » sur la forme de cette 
langue, il nous en représente le moment d'émergence et d'arbitraire. En sorte que le coup double de l'événement tient dans cette réciprocité qui fait survenir la production d'un sens discursif par une re-présentation de la langue. Qui associe une puissance de désignation à une ré-ouverture des écarts de la langue. fois comme un temps et comme un espace (un lieu) particulier : circonscrit, éphémère et dérangeant. La pensée sociologique et urbanistique de l'interstice (Pascal Nicolas-Le Strat), associée à l'approche stylistique et énonciative du figural (Laurent Jenny) permet de redéfinir la traduction non plus comme un transfert linguistique mais comme un frayage énonciatif qui remet en évidence le bricolage des pratiques discursives - et la puissance du potentiel qui travaille le réel dès que nous acceptons de le voir à l'œuvre. Concevoir la traduction comme un «interstice figural» n'est pas une manière d'en faire une image-nous avons appris de Deleuze et Guattari (1980: 89) à nous méfier des métaphores $^{6}$. À nos yeux c'est comme pratique révélant d'autres pratiques que la traduction artiviste opère en / la ville (là où « la langue » veut souvent feindre d'être une entité ou une essence): elle dénaturalise et dés-essentialise au point de révéler la face cachée d'un réel habité de potentialités latentes.

bénéficier du travail de redéfinition théorique d'un outil comme « traduire » pour envisager ce dans quoi nous sommes engagées sous un angle différent? Dans le dernier temps de cette réflexion, nous nous sommes demandé s'il était possible de prendre appui sur cette nouvelle compréhension de la traduction pour mieux comprendre certaines pratiques que nous mettons nous-mêmes en œuvre et, qui semblent, à première vue, échapper à nos protocoles de recherche et à nos grilles d'analyse.

\section{Traduction (géo)graphique de l'hypothèse de la traduction (Sarah Mekdjian)}

Que peut bien vouloir dire «droit à la ville » ? [...] Le droit à la ville ne se réduit [...] pas à un droit d'accès individuel aux ressources incarnées par la ville : c'est un droit à nous changer nous-mêmes en changeant la ville. [...] Mais c'est en outre un droit collectif plus qu'individuel, puisque, pour changer la ville, il faut nécessairement exercer un pouvoir collectif sur les processus d'urbanisation. (Harvey $2011: 8-9$ )

Dans cette dernière partie de notre réflexion croisée, je partirai d'une expérience qui m'a impliquée en tant que chercheure tout en décalant ma posture de géographe - d'où l'appellation bricolée de géographiste. Je m'interroge sur l'utilisation du terme de « traduction » pour penser ce qui s'est joué en pratique dans cette expérience.

Artistes et géographes avons ouvert des ateliers expérimentaux et participatifs de cartographie à Grenoble entre mai et juin 2013 avec des personnes prises dans des situations sociales, géographiques et administratives complexes, la plupart en lutte pour obtenir le droit d'asile en France. L'expérience de ces ateliers a constitué un essai de réinvestissement de la ville de Grenoble, avec des personnes dont le droit de séjour n'était que temporaire ou remis en cause, depuis le geste cartographique. Pendant deux mois, deux fois par semaine, nous nous sommes rencontrés, artistes, géographes, participant.e.s, dans les locaux de l'association Accueil demandeurs d'asile pour créer des cartes d'itinéraires migratoires et urbains. Marie Moreau, Lauriane Houbey et moi-même avons travaillé avec les participant.e.s à partir de plusieurs dispositifs créatifs. Marie 
Moreau a proposé aux participant.e.s de dessiner au feutre noir sur de grandes nappes blanches les parcours jusqu'à Grenoble ; Lauriane Houbey a posé la question, en anglais et en français, des sons entendus pendant les voyages et enregistré les récits des participant.e.s ; j'ai pour ma part proposé de constituer une légende commune à l'aide de gommettes de couleurs, à partir de discussions sur les voyages passés et la vie actuelle à Grenoble. Une fois la légende constituée, chacun.e a dessiné et collé les gommettes au gré de tracés cartographiques individuels.

\section{Fig. 16. Guide cartographique de Grenoble pour les nouveaux arrivants, ateliers de cartographie}

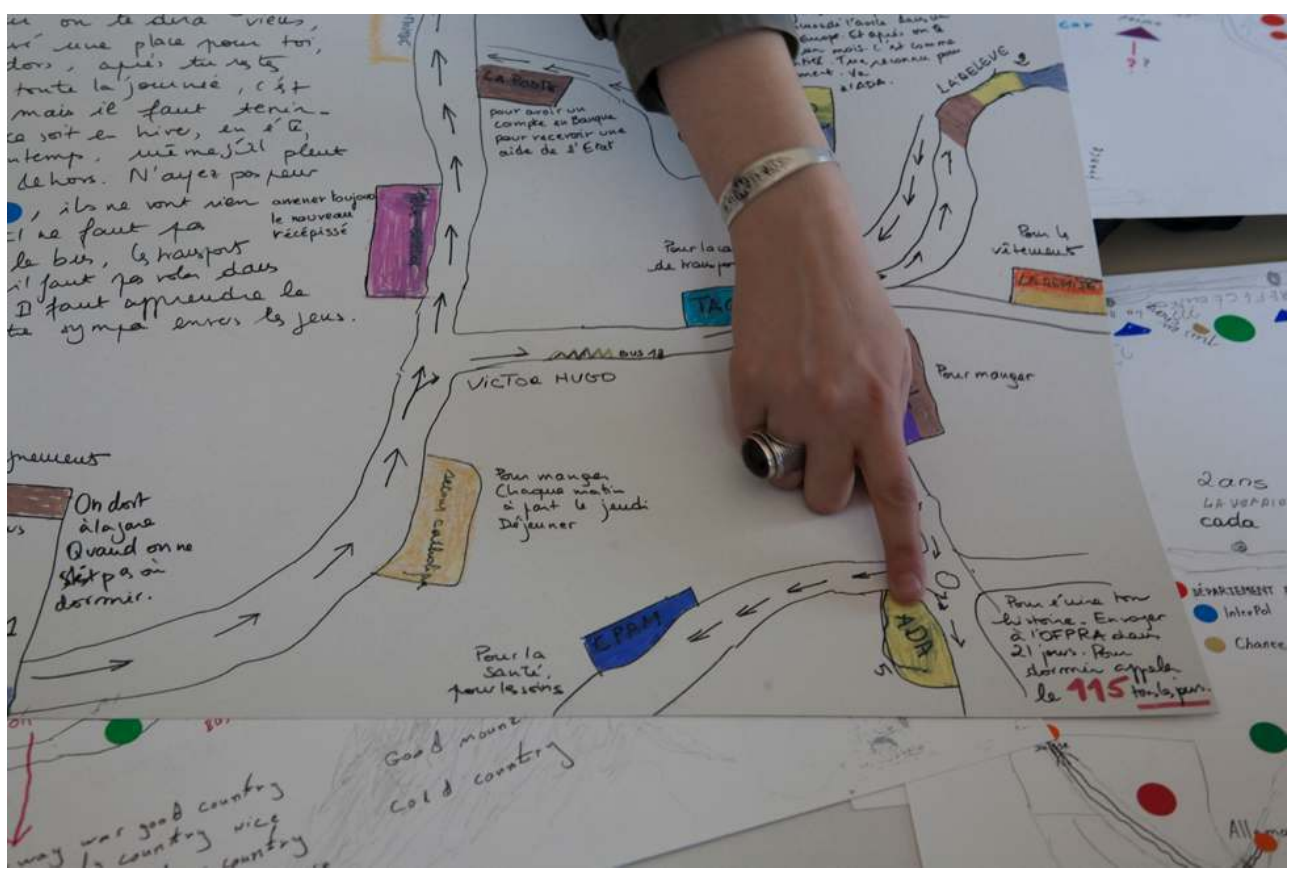

(C) Mabeye Deme. 
Fig. 17. Détail du Guide cartographique de Grenoble pour les nouveaux arrivants, ateliers de cartographie

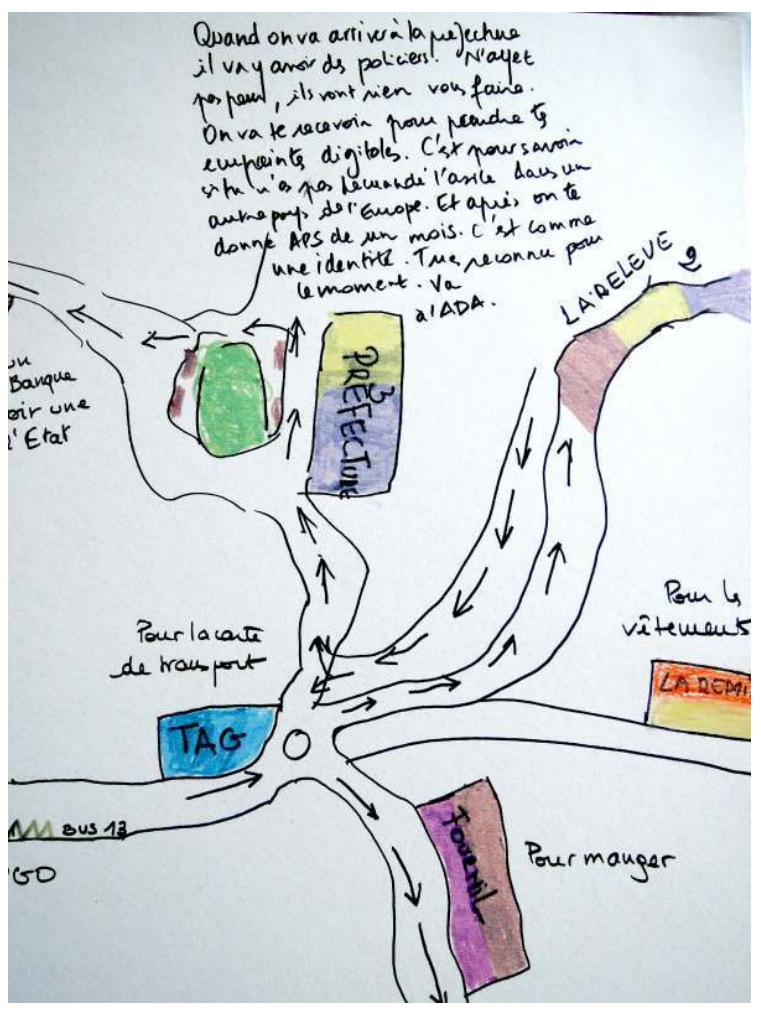

(c) Mabeye Deme.

Le guide cartographique, photographié ci-dessus par Mabeye Deme, a été dessiné par deux participants des ateliers en situation de demande d'asile à l'intention des futurs arrivants. C'est un outil destiné à aider les personnes qui ne sont pas accueillies en France pour vivre et obtenir des droits. À la différence d'un guide, qui trace des itinéraires dans une ville préexistante aux pratiques des citadins, la carte ne permet pas de se repérer de manière stable dans Grenoble. On ne peut se fier ni à l'échelle, ni à la localisation relative des éléments cartographiés les uns par rapport aux autres, mais donne un certain nombre d'indications pratiques sur les lieux-clés à fréquenter et les attitudes sociales à adopter.

L'hypothèse de la traduction se pose, en certain sens, d'elle-même. Les participant.e.s des ateliers parlaient des dizaines de langues différentes, ont habité et parcouru de très nombreux pays, ont été ou sont encore de nationalité soudanaise, érythréenne, arménienne, congolaise, guinéenne, algérienne, azérie et afghane. Certain.e.s étaient à l'époque de nos rencontres demandeur.se.s d'asile, réfugié.e.s, d'autres, exclu.e.s des statuts et circuits administratifs. Les écarts entre les participant.e.s, artistes et chercheurs compris, étaient d'ordre linguistique, discursif, social, administratif, etc. En outre, il est possible de considérer que tracer des cartes a constitué un double acte de traduction. Premièrement, la cartographie nous a permis de créer un cadre d'échanges qui dénaturalisait les rapports de chacun.e à la langue et au discours administratif attendu par les institutions en charge du droit d'asile (Fassin et Kobelinsky 2012). Deuxièmement, les cartes produites, notamment de Grenoble, présentées à travers plusieurs temps d'expositions dans la ville, ont entraîné des questionnements critiques sur les rapports des auteurs et lecteurs des cartes aux espaces urbains qu'ils pratiquent 
quotidiennement. Alors que les administrations fondent leur décision d'attribution du droit d'asile sur "l'interprétation du récit des réfugiés en termes de conformité et de déviance [...] et sur une objectivation de la vérité et du mensonge » (Rousseau et Foxen 2006: 505), les cartes produites ne répondent à aucune injonction de vérité ou de référentialité. Elles ne sont ni « vraies » ni «fausses ». Elles permettent d'exprimer, dans le cadre singulier des ateliers, des bribes de souvenirs, des récits d'affects et d'imaginaires, des revendications, des discours. Au service des relations tissées pendant les ateliers, les cartes résistent à un point de vue surplombant unique et totalisant. Actes de traduction, les cartes sont à la fois des outils qui dénaturalisent nos rapports linguistiques et nos rapports à l'espace urbain. La traduction ici ne consiste pas à transformer la ville de Grenoble telle qu'elle est figurée sur les cartes conventionnelles en un autre référent, tout aussi stable : la ville de Grenoble telle qu'elle est figurée par les personnes en situation de demande d'asile. L'acte de traduction opère autrement. La pratique cartographique est un acte de traduction, au sens où elle produit une carte-guide qui invite aux déplacements (se déplacer par l'imaginaire dans la ville figurée par la carte et se déplacer physiquement dans la ville en abandonnant la recherche de repères stables). Le guide donne la clé d'un ensemble d'attitudes générales à adopter en ville (« ne pas avoir peur des policiers » par exemple) et fait référence à plusieurs lieux-ressources, disposés le long d'un circuit imaginaire.

52 Avec les participant.e.s des ateliers, confrontée.es aux turbulences d'une ville et d'un pays où chacun.e était si peu accueilli.e, nous avons tenté de cartographier les lieux de refuge, à inventer et à soutenir. Marie Moreau, dans un entretien que j'ai mené avec elle en 2014, parle de la constitution en plusieurs temps et plusieurs lieux d'un atlas local non exhaustif, référentiel et non référentiel, anticonventionnel. Cet atlas est en mouvement, jamais complété :

Les cartes produites ouvrent des brèches. Par ces brèches viennent des récits inédits. Parfois, d'une conversation à l'autre, deux cartes tout à fait différentes ont été dessinées par la même main. Celles et ceux qui emploient la carte pour « représenter » (se représenter) s'en servent pour diverses raisons. Elles inventent un monde qui n'existait pas avant ou qui n'existe pas en réalité. Elle figure et configure une relation à l'espace à un moment donné. Cette relation est relative et évolue. C'est pour cela que ces cartes s'exposent, se colportent, Les cartes d'Atlaslocal sont encore en cours.

Pour Lauriane Houbey :

Les espaces-ressources sont des imaginaires-ressources. Des environnements où tout n'est pas encore tracé, écrit, ou bien des zones où je sens que d'autres personnes en ont d'autres usages que moi, ou des zones qui n'ont peut-être pas d'usage, nonaffectées. Non-affectées au sens fonctionnel comme au sens esthétique du terme. (Houbey et Moreau $2012: 2$ )

Sans doute rejoignons-nous là l'idée de re-potentialisation : il ne s'agit pas tant de jouer une réalité contre une autre réalité que de permettre d'appréhender le « réel » comme potentialité. Nous touchons aussi au point où les artistes se saisissent des codes géographiques pour les faire fonctionner selon d'autres logiques.

De plus en plus de collectifs composés d'artivistes et de géographes se constituent, autour de la question de la (contre)cartographie en tant que pratique critique et créative, pour proposer des alternatives aux cartes utilisées classiquement au service du contrôle et de la traçabilité des individus, de la gouvernementalité des populations et de la planification de la ville néolibérale. The Counter-Cartographies Collective souhaite élaborer une 
pratique cartographique qui «crée de nouvelles possibilités (de géographie politique) et d'autres réalités (de géographie politique), plutôt qu'elle ne représente des géographies déjà existantes " (Dalton et Mason-Deese 2012: 443). La carte, qui est «toujours par rapport à son référent dans un écart que rien ne peut réduire et qui en est constitutif » (Tiberghien 2010: 198), configure des mondes qui ne préexistaient pas à l'acte de cartographie et qui viennent transformer nos autres sources d'expériences sociospatiales. Les artistes contemporains se saisissent de la pratique cartographique non pas pour «combler cet écart mais [...] y circuler» (Tiberghien 2010: 199), le creuser, lui donner des formes nouvelles. Principalement à partir des années 1960, la pratique cartographique est mobilisée précisément pour critiquer l'illusion d'une référentialité objective et positiviste des cartes. Guy Debord et les situationnistes inventent un « jeu de cartes ", qui s'inspire des écrits de Henri Lefebvre, en particulier de La Critique de la vie quotidienne parue en $1947^{7}$. Henri Lefebvre invite à pratiquer la ville en « joueur ", pour prendre conscience des règles du jeu imposées par l'urbanisme moderniste et naturalisées par les cartes, et "se jouer ", à proprement parler, de ces conventions. La pratique situationniste de la dérive, élaborée par Guy Debord, propose de perdre ses repères pour un temps. Voici les règles du jeu à adopter :

Une ou plusieurs personnes se livrant à la dérive renoncent, pour une durée plus ou moins longue, aux raisons de se déplacer et d'agir qu'elles se connaissent généralement, aux relations, aux travaux et aux loisirs qui leur sont propres, pour se laisser aller aux sollicitations du terrain et des rencontres qui y correspondent. [...] La part de l'exploration [...] est minime, par rapport à celle d'un comportement déroutant, dans le "rendez-vous possible ». Le sujet est prié de se rendre seul à une heure qui est précisée dans un endroit qu'on lui fixe. Il est affranchi des pénibles obligations du rendez-vous ordinaire, puisqu'il n'a personne à attendre. Cependant ce «rendez-vous possible" l'ayant mené à l'improviste en un lieu qu'il peut connaître ou ignorer, il en observe les alentours. [...] Les enseignements de la dérive permettent d'établir les premiers relevés des articulations psychogéographiques d'une cité moderne. Au-delà de la reconnaissance d'unités d'ambiances, de leurs composantes principales et de leur localisation spatiale, on perçoit les axes principaux de passage, leurs sorties et leurs défenses. On en vient à l'hypothèse centrale de l'existence de plaques tournantes psychogéographiques. On mesure les distances qui séparent effectivement deux régions d'une ville, et qui sont sans commune mesure avec ce qu'une vision approximative d'un plan pouvait faire croire. On peut dresser, à l'aide des vieilles cartes, de vues photographiques aériennes et de dérives expérimentales une cartographie influentielle qui manquait jusqu'à présent, et dont l'incertitude actuelle, inévitable avant qu'un immense travail ne soit accompli, n'est pas pire que celle des premiers portulans, à cette différence près qu'il ne s'agit plus de délimiter précisément des continents durables, mais de changer l'architecture et l'urbanisme. (Debord 1956)

La dérive crée les conditions d'une " déroute ", d'une perte de repères, à partir desquelles s'élaborent des cartes destinées à refigurer, de manière temporaire, les espaces urbains traversés.

Nombreux sont les artistes-marcheurs / arpenteurs qui pratiquent la marche, souvent en produisant des cartographies artistiques et artivistes (O'Rourke 2013). Mathias Poisson, artiste promeneur, réalise des «graphies du déplacement» (Poisson 2010) et passe des journées :

[...] à marcher dans des villes inconnues, à ausculter des quartiers, à explorer des zones marginales, à trouver des échappées, à musarder sous les ponts, dans des bois, et à contempler les paysages qui sont là. [...] Je témoigne de sensations, de préoccupations et d'observations survenues lors de ces déambulations hasardeuses. 
Je dessine des cartes qui décrivent ce que j'ai vécu ou je dessine des cartes qui proposent d'accomplir des marches possibles sur des territoires que j'ai arpentés. [...] Je laisse apparaître ce que le geste et le souvenir me restituent. Le dessin fait le récit d'une déambulation, grossissant certains éléments perçus, omettant d'autres, déformant les points de vue. (Poisson 2010 : 105).

Fig. 18. Carte de promenades périlleuses dans les paradis infernaux de Corbières, Mathias Poisson

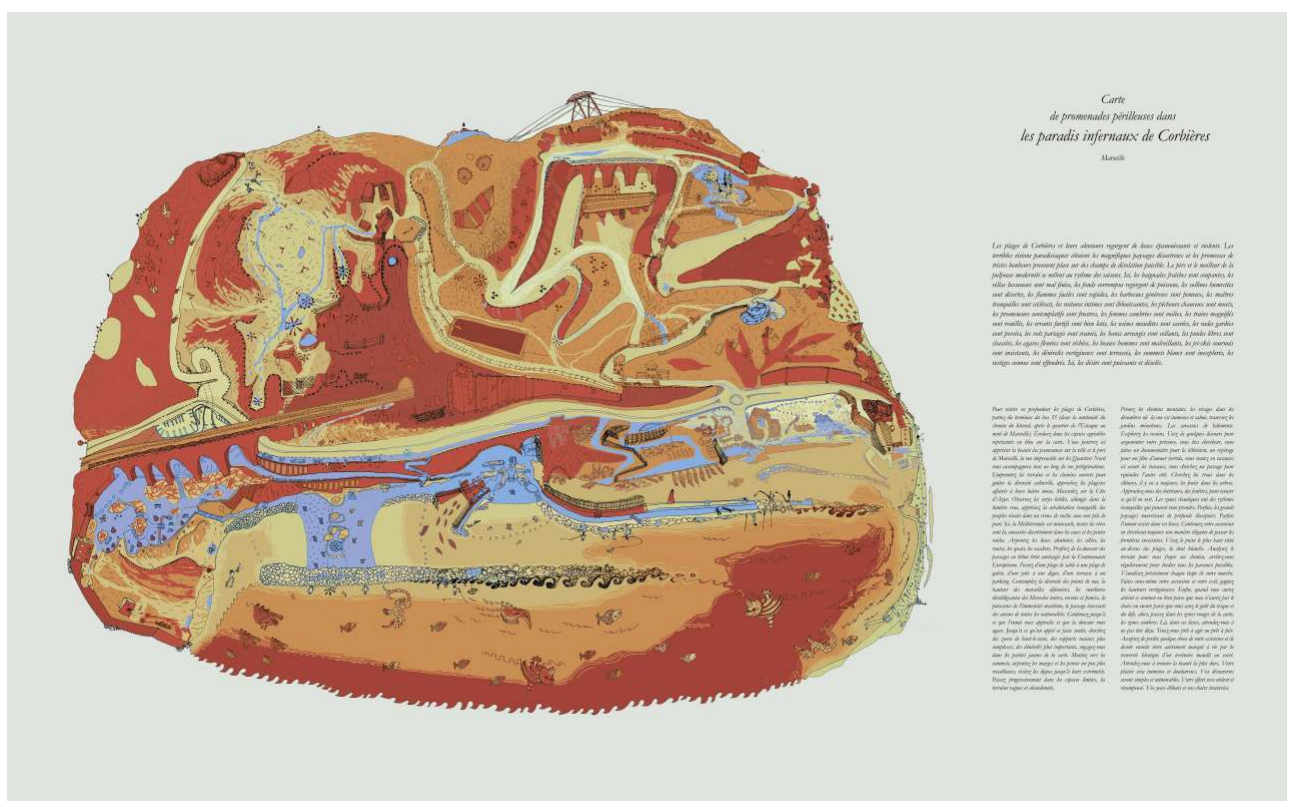

(c) Les Corbières, Mathias Poisson

58 De Mathias Poisson, soulignons encore qu'il présente lui-même sa démarche artistique comme une « indiscipline liquide » puisqu'il utilise dessin, texte, danse et arts plastiques " comme des vases communicants ${ }^{8} »$. Explorant aussi la ville par la marche, Lauriane Houbey, artiste chorégraphe, a réalisé avec Marie Moreau, artiste plasticienne, l'exposition Géographies intérieures à Grenoble en 2012, à partir de relevés cartographiques tracés en mouvement. Lauriane Houbey explique dans un entretien mené en 2012, avoir apporté des carnets à dessins lors de maraudes qu'elle effectuait dans la ville de Grenoble:

La Maraude Cartographique se dote de carnets à spirale, format A4 $(21 \times 29 \mathrm{~cm})$, et de feutres plus ou moins épais. Le matériel tient dans un sac à dos, puis se pose sur les genoux ou à même le sol, sur le trottoir, sur une marche. Les carnets sont parfois mis bout à bout, côte à côte, pour permettre au cadre de se déployer avec le récit. Ils les arrêtent parfois, parce que tourner la page, retomber sur une page blanche, ça demande de re-solliciter son énergie. Alors on prend rendez-vous pour une autre fois. Parfois on ne se revoit pas.

Marie Moreau explique son intérêt pour la cartographie expérimentale urbaine en relation à un désenchantement (Houbey et Moreau $2012: 2$ ) :

Au début, il y a le désir de réaliser des travaux qui s'articulent à des lieux d'accueil - sanitaires et sociaux - qui ont une histoire et une action qui m'importent à Grenoble. Il y a aussi ces différentes collaborations avec Lauriane autour du paysage, du territoire grenoblois. Et cette histoire commune, celle des occupations d'espaces - squats -, qui s'est soldée par une chronique d'expulsions. Une opération de mise sous silence. Et qui s'opère aujourd'hui encore. La ville m'est alors apparue comme une coquille creuse. En tout cas, c'est comme ça, un temps, que je l'ai vécu. Désenchantée. J'ai eu besoin de trouver des espaces-ressources, comme des restes 
de terrains vagues, des passages, des zones non exploitées où il est encore possible

de flâner, de se perdre, de rêver.

60 À partir de pratiques créatives distinctes, Lauriane Houbey, Marie Moreau et Mathias Poisson mobilisent le geste cartographique comme support d'un récit qui se construit «chemin faisant ». Le chemin emprunté n'est pas linéaire ou déjà tracé sur une carte conventionnelle, mais fait de tours, de détours, de pages remplies et de pages blanches. Ces deux artistes s'intéressent particulièrement aux espaces des marges, aux interstices de la ville: dents creuses, berges fluviales, «lieux étonnants ${ }^{9}$ ", qui subvertissent les vitrines et les paysages urbains dominants. Les cartes dessinées impliquent la participation de citadins rencontrés sur les chemins tracés, avec lesquels les artistes choisissent de marcher. Ces déambulations sensibles engagent autant une dimension individuelle qu'une dimension collective de l'expérience urbaine. La cartographie sert ici à ouvrir des brèches dans les cartes et plans conventionnels des villes parcourues.

61 Les cartes constituées chemin faisant sont proches des cartes d'itinéraires, que Jean-Marc Besse propose de créer et qui «fourni[ssent] une représentation du territoire dans laquelle celui-ci n'est pas considéré indépendamment des pratiques qui s'y déploient [...] mais au contraire défini dans sa structure même par les engagements pratiques de ceux qui y inscrivent leurs déambulations " (Besse 2010: 7). Avec les cartes d'itinéraires, on pourrait également citer les vidéocartographies de Bouchra Khalili sur des itinéraires migratoires vers l'Europe ou encore de Till Roeskens sur la construction et l'organisation d'un camp de réfugiés palestiniens en Cisjordanie, qui montrent les traits de dessin en train d'être tracés, au rythme du récit oral fait par les migrants et habitants dessinateurs, au gré du cheminement de la mémoire et des affects. La carte en traduction place son utilisateur dans un état instable où précisément aucun chemin n'est tracé a priori.

Une certaine géographie critique n'est pas si loin de ces pratiques artivistes. Le «droit à la ville » défini par Henri Lefebvre (1968) se construit dans ces actes infimes, qui opèrent par déplacements, étrangements, battements, au cœur des espaces que nous pratiquons, habitons, traversons. La (contre)cartographie est une des pratiques possibles de réinvention de nos quotidiens urbains. Quel est le potentiel critique et agissant de ces interventions entre art et activisme politique, alors que l'art en ville sert si souvent des processus de marchandisation de l'espace urbain? Une critique des modes capitalistes d'urbanisation, dans l'optique d'une transformation de nos vies citadines quotidiennes, est-elle possible par l'art et l'imaginaire?

Les aménageurs et urbanistes, qui travaillent à la compétitivité des villes au sein de la mondialisation néolibérale, intègrent de plus en plus l'art dans leurs projets de rénovation et de réhabilitation urbaines. L'art s'invite dans les espaces-vitrines des métropoles en tant que faire-valoir de processus d'accumulation du capital, par ailleurs facteur de dépossession, de privatisation de l'espace public et d'accroissement des inégalités socio-spatiales (Gintrac et Giroud 2014).

Dans son ouvrage intitule The Rise of the Creative Class. And How It's Transforming Work, Leisure and Everyday Life, paru en 2002, Richard Florida - directeur et professeur de commerce et créativité à l'Institut Martin Prosperity de l'université de Toronto - propose de transformer la ville fordiste en ville créative. Cet ouvrage peut servir à la fois de mise en place et de repoussoir pour une pensée géographique de la ville contemporaine à travers la prise de l'art. Expliquant l'émergence d'une "classe créative », au service d'un modèle de "ville créative", fondée sur les pratiques artistiques et culturelles, l'auteur défend la nécessaire multiplication des politiques culturelles en ville pour attirer les 
individus appartenant à cette classe socio-culturelle, soit les artistes, les journalistes, les artisans ou encore les travailleurs du secteur des hautes technologies. La présence des "créatifs» renforcerait l'offre culturelle des villes et serait, de ce fait, la clé d'un renouveau économique des métropoles post-industrielles. De nombreuses municipalités se sont clairement inspirées des travaux de Richard Florida, pour transformer leurs images de «villes en crise » en "villes créatives » à nouveau compétitives ${ }^{10}$. L'art et la culture sont utilisés en tant qu'outils économiques au service de l'image commerciale et de la compétitivité économique des villes.

Loin de déstabiliser le capitalisme urbain contemporain, la théorie développée par Richard Florida vise à adapter les modalités d'accumulation du capital, de production de plus-value et de mise en concurrence des villes à la crise du fordisme. Comme l'explique l'argumentaire du colloque qui s'est tenu à Grenoble en 2013 sur l'esthétisation de l'espace public :

Les recompositions territoriales contemporaines s'appuient sur des politiques d'image qui empruntent à l'art et à la culture leur puissance symbolique. [...] Les processus de restructuration des systèmes productifs fordistes ont placé l'économie culturelle et les industries créatives au centre des débats sur l'avenir des territoires.

C'est tout l'inverse dont il est question avec les interventions artivistes analysées plus haut.

L'artivisme urbain ne se contente pas de passer d'un modèle urbain à un autre (de la ville fordiste à la ville créative) sans remise en cause des référents existants. Plutôt que d'envisager la traduction comme le passage d'un modèle de ville à un autre, l'hypothèse développée ici vise plutôt à considérer la traduction en tant que pratique continue, sinon permanente-en tout cas non accidentelle. Les propositions artivistes créent des modalités de pratiques urbaines selon lesquelles les citadins peuvent en permanence interroger et déstabiliser leur environnement. Cette posture active et critique rompt avec les visions totalisantes et surplombantes de la ville voulue compétitive produites par les experts en urbanisme, qui peinent à inclure les usagers dans l'élaboration de leurs projets. L'hypothèse de la traduction, envisagée comme un outil de réappropriation de la ville depuis ses usages et usagers ordinaires, fait signe vers les écrits de Michel de Certeau sur la marche. Celle-ci est définie en tant que pratique de transformation du rapport des citadins à la ville depuis le sol, et non depuis la vision surplombante et techniciste de la ville, propre aux urbanistes, architectes et aménageurs (Certeau 1990). L'acte de traduction désigne précisément ces efforts de déconstruction mis en acte par les citadins dans le quotidien urbain, plutôt que de construction d'autres modèles totalisants de villes.

\section{Conclusion}

La reconception ou reconfiguration respective de "la ville " et de "la traduction", opérée ici de manière polyphonique en prenant appui sur des cas d'artivisme, semble mener à une mise en avant des pratiques. La traduction aura ici servi à penser les écarts, marges, intervalles, interstices et autres bougés dans de la ville. «Traduire » aura été le nom donné à chaque entreprise de déstabilisation des certitudes et se sera, en retour, redéfini comme une manière de re-potentialiser l'existant: l'espace-temps d'un battement, le « réel » vacille et redevient une simple facette aléatoire d'un possible. Nous avons donc employé «traduction » comme un terme-outil pour un temps : le temps où il 
peut être efficace pour penser autrement. Lorsque son potentiel critique sera émoussé, il faudra en forger un autre.

Sans chercher à aboutir à des conclusions définitives, nous prenons acte de la nécessité d'engager notre responsabilité en tant que chercheures pour rejoindre les initiatives qui font de la pratique de traduction un interstice où l'imaginaire d'alternatives communes redevient possible. Engager le dialogue au lieu de se poser en expertes constituait à nos yeux un pas dans cette direction. Espérons que la mise en avant de l'amitié, trop souvent masquée par l'apparente neutralité objective du discours scientifique, saura diffuser et essaimer suffisamment pour donner lieu et suite à des réponses multiples. Pour prolonger cette expérimentation et la rendre interactive, nous vous invitons à participer à la version interactive disponible à cette adresse, où vous pouvez verser vos propres découvertes et contributions. Nous avons hâte à vous lire!

\section{BIBLIOGRAPHIE}

Ackan, Esra, 2012, Architecture in Translation: Germany, Turkey and the Modern House, Durham, Londres, Duke University Press.

Anderson, Benedict, 1983, Imagined Communities: Reflections on the Origin and Spread of Nationalism, Londres, New York, Verso.

Augaitis, Daina, 2011, Muntadas: Entre/between, Madrid, Museo nacional centro de arte Reina Sofía.

Augé, Marc, 1992, Non-lieux. Introduction à une anthropologie de la surmodemité, Paris, Seuil, coll. « La Librairie du $\mathrm{xx}^{\mathrm{e}}$ siècle ».

Augoyard, Jean-François, 2000, « L'action artistique dans l'espace urbain », dans J. Métral (dir.), Cultures en ville ou de l'art et du citadin, Paris, Éditions de l'Aube, p. 14-23.

Baudrillard, Jean, 1976, Kool killer ou l'insurrection par les signes, Paris, Les Partisans du moindre effort, [En ligne], http://lpdme.org/projects/jeanbaudrillard/koolkiller.pdf, consulté le 10 avril 2015.

Besse, Jean-Marc (dir.), 2010, Les Carnets du paysage, $\mathrm{n}^{\circ}$ 20, « Cartographies ».

Bhabha, Homi, 2006, « Le Tiers-espace. Entretien avec Jonathan Rutherford », traduction de l'anglais par Christophe Degoutin et Jérôme Vidal, Multitudes, $n^{\circ} 26$, [En ligne], http:// www.multitudes.net/Le-Tiers-espace-Entretien-avec/, consulté le 3 février 2015.

Certeau, Michel de, 1990, L'Invention du quotidien. Tome 1: Arts de faire, Paris, Gallimard.

Dalton, Craig et Mason-Deese, Liz, 2012, « Counter (Mapping) Actions: Mapping as Militant Research », ACME: An International E-Journal for Critical Geographies, vol. 11, n 3, p. 439-466, [En ligne], http://ojs.unbc.ca/index.php/acme/article/view/941, consulté le 3 février 2015.

Debord, Guy, [1956] 2011, « Théorie de la dérive », La Revue des ressources, [En ligne], http:// www.larevuedesressources.org/theorie-de-la-derive,038.html, consulté le 3 février 2015.

Deleuze, Gilles et Guattari, Félix, 1980, Mille Plateaux. Capitalisme et schizophrénie, Paris, Minuit. 
Douay, Nicolas, 2012, «L'activisme urbain à Montréal : des luttes urbaines à la revendication d'une ville artistique, durable et collaborative ", L'Information géographique, n 76, p. 83-96.

Ducharme, Francis, 2009, L'Imaginaire de l'espace urbain montréalais dans Bienvenue à et Ascension, deux déambulatoires audioguidés d'Olivier Choinière, Mémoire de l'UQAM, sous la supervision de Shawn Huffman et Lucie Robert, [En ligne], http://www.archipel.uqam.ca/1944/1/M10791.pdf.

Fassin, Didier et Kobelinsky, Carolina, 2012, « Comment on juge l'asile. L'institution comme agent moral », Revue française de sociologie, vol. 53, n 4, p. 657-688.

Garnier, Jean-Pierre, 2011, «La "classe créative" : un nouveau mythe urbain pour de nouvelles mystifications urbanistiques », Terrains de luttes, [En ligne], http://terrainsdeluttes.ouvaton.org/? p=447, consulté le 10 avril 2015.

Gintrac, Cécile et Giroud, Matthieu (dir.), 2014, Villes contestées. Pour une géographie critique de l'urbain, Paris, Les Prairies Ordinaires.

Harvey, David, 2011, Le Capitalisme contre le droit à la ville, traduction Cyril Le Roy et Nicolas Vieillescazes, Paris, Éditions Amsterdam.

Harvey, David, 2012, Rebel Cities: From the Right to the City to the Urban Revolution, Londres, Brooklyn, Verso.

Hatzfeld, Hélène et Marc, Ringart, Nadja, 1998, Quand la marge est créatrice, les interstices urbains initiateurs d'emploi, Paris, Éditions de l'Aube.

Hocquard, Emmanuel, 1997, « Taches blanches », dans Le Gam, http://wings.buffalo.edu/epc/ orgs/bureau/tb_f.html, consulté le 10 mai 2016. Repris dans Ma Haie. Un privé à Tanger II, Paris, P.O.L., 2001.

Hou, Jeffrey, 2010, Insurgent Public Space: Guerrilla Urbanism and the Remaking of Contemporary Cities, New York, Routledge.

Houbey, Lauriane et Moreau, Marie, 2012, Géographies intérieures. Document d'artiste, Grenoble, Laboratoire d'Art Aujourd'hui, [En ligne], http://www.journee-art-contemporain.com/wpcontent/uploads/2012/09/Dossier_presentation_Laa.pdf, consulté le 3 février 2015.

Jenny, Laurent, 1990, La Parole singulière, Paris, Belin, coll. « L'extrême contemporain ».

Landow, George, 2006, Hypertext 3.0, Baltimore, John Hopkins University.

Lefebvre, Henri, [1968] 2009, Le Droit à la ville, Paris, Economica.

Lévy, Pierre, 1998, Qu'est-ce que le virtuel ?, Paris, La Découverte, [En ligne], http:// hypermedia.univ-paris8.fr/pierre/virtuel/virt0.htm, consulté le 10 mai 2016.

Lindgaard, Jade, 2005, Vacarme, $\mathrm{n}^{\circ}$ 31, « Artivisme ».

Meschonnic, Henri, 2000, Et le génie des langues?, Vincennes, Presses universitaires de Vincennes, coll. « Essais et savoirs».

Meschonnic, Henri, 1997, De la langue française : essai sur une clarté obscure, Hachette, Paris.

Nicolas-Le Strat, Pascal, 2007, « Multiplicité interstitielle », Multitudes, $\mathrm{n}^{\circ} 31$, [En ligne], http:// www.cairn.info/article.php?ID_ARTICLE=MULT_031_0115, consulté le 10 avril 2015.

O’Rourke, Karen, 2013, Walking and Mapping: Artists As Cartographers, Cambridge, MA, MIT Press.

Paquot, Thierry, 2010, « Le jeu de cartes des situationnistes », CFC, $\mathrm{n}^{\circ}$ 204, [En ligne], www.lecfc.fr/new/articles/204-article-7.pdf.

Poisson, Mathias, 2010, « Graphie du déplacement », Les Carnets du Paysage, n 20, p. 104-115. 
Reynolds, Richard, 2008, On Guerrilla Gardening: A Handbook for Gardening Without Boundaries, New York, Bloomsbury.

Rousseau, Cécile et Foxen, Patricia, 2006, « Le mythe du réfugié menteur : un mensonge indispensable ", L'Évolution psychiatrique, $\mathrm{n}^{\circ}$ 71, p. 505-520.

Simay, Philippe, 2008, "Une autre ville pour une autre vie. Henri Lefebvre et les situationnistes », Métropoles, $n^{\circ} 4$, [En ligne], http://metropoles.revues.org/2902.

Simon, Sherry, 2007, Translating Montreal: Episodes in the Life of a Divided City, Montréal, Mcgill Queens University Press.

Simon, Sherry, 2008, Traverser Montréal : une histoire culturelle par la traduction, traduit de l'anglais par Pierrot Lambert, Montréal, Fides.

Tiberghien, Gilles A., 2010, « Poétique et rhétorique de la carte dans l'art contemporain », L'Espace géographique, vol. 39, $\mathrm{n}^{\circ}$ 3, [En ligne], www.cairn.info/revue-espace-geographique-2010-3page-197.htm, consulté le 3 février 2015.

Young, James E., 1993, The Texture of Memory: Holocaust Memorials and Meaning, New Haven, Yale University.

\section{NOTES}

1. Il faut noter que l'entrée «traduction » s'avère parfois être un piège car elle renseigne aussi la mise en français, pour les besoins de ce site francophone, d'une œuvre où figure un texte en anglais et qui, à première vue, n'engage aucun type de rapport traductif. Notons en outre que les résultats obtenus sont différents si l'on cherche «translation » dans l'interface en anglais (aucun résultat avec «translating»). Enfin, il faut mentionner un résultat lorsque l'on cherche «translation » dans l'interface en français : il s'agit d'une sculpture de Jacqueline Metz et Nancy Chew intitulée Arboreus, after Rodchenko, et installée en 2007 au Lynn Valley Library \& Plaza de Vancouver. L'œuvre se compose d'une photographie, qui reprend en le modifiant le Pine Tree d'Alexander Rodchenko - $\mathrm{y}$ insérant, notamment, l'inscription du nom du cèdre en latin «Cupressaceae Thuja plicata » - et d'une seconde inscription : une citation de Borges traduite en anglais : "The original is unfaithful to the translation", suivie du nom de l'auteur : "Jorge Luis Borges », cf. http://plepuc.org/fr/oeuvre/arboreus-after-rodchenko et http:// www.metzchew.com/galleries/arboreus/arboreus.html (consulté le 10 mai 2016).

2. La référence précise est Terry Fox, Hobo Signs: A Compilation of Signs for Those who May One Day Find Them Useful, Munich, Kunstraum, 1985.

3. Sur son site, l'artiste évoque une déclinaison portative de l'œuvre sur un t-shirt dont la face reprend le texte en braille mais en deux dimensions, le rendant indéchiffrable tandis que le tableau de Snellen figure, au dos, mais dans la même couleur blanche que le tissu, si bien qu'on ne peut pas le lire non plus.

4. En Europe, on peut penser aux « contre-monuments " comme le Disappearing Monument Against Fascism and War installé en 1986 par Jochen et Esther Shalev-Gertz's à Harburg, en Allemagne, stèle de 12 mètres de haut peu à peu amenuisée jusqu'à disparaitre totalement (Young 1993).

5. http://www.sacreemontagne.onf.ca/\#/sacreemontagne; le site n'étant malheureusement plus navigable, une archive se trouve ici: http://nt2.uqam.ca/fr/repertoire/sacree-montagne-0 (consulté le 10 avril 2015).

6. De ce fait, notre démarche diffère radicalement de celle d'Homi Bhabha (2005), même si le résultat peut sembler proche. 
7. Rappelons que les situationnistes pratiquaient la dérive en flânant jusqu'à se perdre, en collant des morceaux de cartes épars ou encore en abordant une ville avec, en main, la carte d'une autre ville (Simay 2008 et Paquot 2010).

8. Mathias Poisson, http://poissom.free.fr/?browse=d\%E9marche\%20artistique (consulté le 3 février 2015).

9. Mathias Poisson, http://poissom.free.fr/?browse=MathiasPoisson (consulté le 3 février 2015).

10. Si le modèle de la «ville créative » connaît un écho certain dans les politiques urbaines et la recherche actuelle en géographie, il est aussi très fortement critiqué. Dans un article intitulé «La "classe créative" : un nouveau mythe urbain pour de nouvelles mystifications urbanistiques ", Jean-Pierre Garnier, sociologue urbain, demande «à quelle "vie" mène cette avant-garde (auto)proclamée ? Et à quelle survie tous ceux qui n'en font pas partie ?» (Garnier 2011).

\section{RÉSUMÉS}

Cet article s'intéresse à des pratiques urbaines entre art et action politique. Installer un potager illégal sur une avenue, détourner les cartes ou les panneaux de signalisation: et si c'était traduire? Notre hypothèse consiste à penser les œuvres et pratiques "artivistes » en tant qu'actes de traduction, en considérant que ces pratiques opèrent par décalages dans l'espace de la ville (plan de l'objet analysé) et en procédant nous-mêmes par traductions (méta-plan de l'analyse). Ce faisant, nous nous positionnons comme hétérolinguiste (Myriam Suchet) et géographiste (Sarah Mekdjian) pour éprouver ce qui se modifie de nos manières de penser lorsque l'on articule recherche, action et création - ce que nous nommons une perspective indisciplinaire. Nous commencerons par établir une typologie ad hoc de quelques œuvres artivistes installées notamment à Montréal pour redéfinir la traduction en termes de battement et d'interstice, avant d'en faire une hypothèse pour analyser des pratiques cartographiques créatives menées à Grenoble en 2013.

This article deals with urban practices which combine art and political action. Can setting up an informal vegetable garden in a street, turning maps or road signs around, etc. be analysed as many forms of translation? Our hypothesis is to think of artivist works and practices as acts of translation by considering that these practices operate displacements within city spaces (plan of the analysed object) and by operating ourselves translations (meta-plan of analysis). In doing so, we become heterolingualist (Myriam Suchet) and geographist (Sarah Mekdjian) in order to experience what changes in our way of thinking when we tie together research, action and creation-what we call an indisciplinary perspective. We start by establishing an ad hoc typology of various artivist installations, principally based in Montreal in order to redefine translation in terms of "battement" and "interstice" before developing it into a hypothesis for the analysis of creative cartographic practices carried out in Grenoble in 2013. 
INDEX

Mots-clés : artivisme, carte, expérience, indiscipline, interstice, pratiques, traduction, urbain, espace, ville

Keywords : artivism, experience, city, indiscipline, interstice, map, practices, space, translation, urban

\section{AUTEURS}

MYRIAM SUCHET

Université Sorbonne Nouvelle - Paris 3, Sorbonne Paris Cité, THALIM (UMR 7172)

\section{SARAH MEKDJIAN}

Université Pierre-Mendès France-Grenoble II, PACTE (UMR 5194) 